\title{
Regio- and Enantioselective Allylic Substitution with Less Active $N$ - or $O$-Nucleophiles Catalyzed by Iridium-Complex of Bis(oxazolinyl)pyridine
}

\author{
Hideto Miyabe, ${ }^{*, a, b}$ Katsuhiko Moriyama, ${ }^{a}$ and Yoshiji Takemoto*,a \\ ${ }^{a}$ Graduate School of Pharmaceutical Sciences, Kyoto University; Yoshida, Sakyo-ku, Kyoto 606-8501, Japan: and ${ }^{b}$ School \\ of Pharmacy, Hyogo University of Health Sciences; Minatojima, Chuo-ku, Kobe 650-8530, Japan. \\ Received January 27, 2011; accepted February 28, 2011
}

The utility of hydroxylamines as nitrogen nucleophiles was investigated in the iridium-catalyzed regio- and enantioselective allylic substitution. Allylic substitution with hydroxylamines proceeded with good enantioselectivities by using the iridium-complex of bis(oxazolinyl)pyridine ligand. The good regio- and enantioselectivities were also achieved in the reaction with alkylamines, $p$-anisidine, and 4-methoxyphenol.

Key words allylic substitution; catalytic; enantioselective; iridium

Enantioselective transition metal-catalyzed allylic substitutions have been developed as fundamentally important crosscoupling reactions. ${ }^{1-8)}$ In general, the heteroatom nucleophiles in these reactions have been largely limited to alkylamines, anilines, carboxylates and phenols. Our laboratory is interested in searching the synthetically useful heteroatom nucleophiles for the synthesis of functionalized allylic compounds (Fig. 1). ${ }^{9)}$ As our successful examples, we have recently reported the utility of oximes $\mathbf{1}$ and guanidines $\mathbf{5}$ and $\mathbf{6}$ as nucleophiles in the transition metal-catalyzed allylic substitution. $^{10-14)}$

Hydroxylamines are also attractive synthetic reagents for allylic substitution, since they have nitrogen and oxygen atoms as nucleophiles. However, the allylic substitution with hydroxylamines has not been studied well and is limited to a simple palladium-catalyzed amination ${ }^{15,16)}$; thus, there are no reports on asymmetric reactions. We have recently reported that hydroxylamines $\mathbf{2}$ and $\mathbf{3}$ having an $\mathrm{N}$-electron-withdrawing substituent, also known as hydroxamic acids, act as reactive oxygen nucleophiles in the enantioselective allylic substitution (Fig. 1) ${ }^{17,18)}$ As a part of our program directed toward searching the synthetically useful heteroatom nucleophiles, we describe in detail the study of hydroxylamines $\mathbf{4}$ as nitrogen nucleophiles in the regio- and enantioselective iridium-catalyzed allylic substitutions. ${ }^{19)}$ In this study, we also expected that comparison with alkylamines, $p$-anisidine, and 4-methoxyphenol would lead to informative suggestions regarding the asymmetric reaction using the iridium complex of pybox (bis(oxazolinyl)pyridine) ligand.

\section{Results and Discussion}

Controlling both regio- and enantioselectivities has been of great importance in the allylic substitution of unsymmetrical substrates with heteroatom nucleophiles. ${ }^{20-24)}$ The regioselectivities in reactions using rhodium, ${ }^{25-33)}$ iridium, ${ }^{34-38)}$ and ruthenium ${ }^{39-41)}$ complexes are quite different from those of palladium-catalyzed reaction. Therefore, chiral iridium complexes controlling regio- and enantioselectivities have been a subject of current interest. ${ }^{42-84)}$ Recently, we reported that the iridium-complex of pybox catalyzed allylic substitution of unsymmetrical substrates to form branched products with good enantioselectivities. ${ }^{13,14,17,19)}$

Prior to exploring the enantioselective reaction, we first investigated the viability of hydroxylamine $\mathbf{4 A}$ having $N$-ben- zoyl and $O$-benzyl groups (Chart 1). Although the reaction of 4A with carbonate 7 was less effective in the absence of a base, the reaction of $\mathbf{4 A}$ with acetate 8 proceeded smoothly by employing $\mathrm{Et}_{2} \mathrm{Zn}$ as a base to give the branched product 9Aa in $60 \%$ yield without formation of the linear product.

Based on these results, we next investigated iridium-catalyzed asymmetric allylic substitution with hydroxylamine $\mathbf{4 A}$ under basic conditions (Chart 2). In this study, phosphate 10a was employed as an unsymmetrical substrate to prove the efficiency of iridium complex of pybox ligand 12. In our preliminary communication, ${ }^{19)}$ we reported that the base drama tically influenced the regio- and enantioselectivities. Here

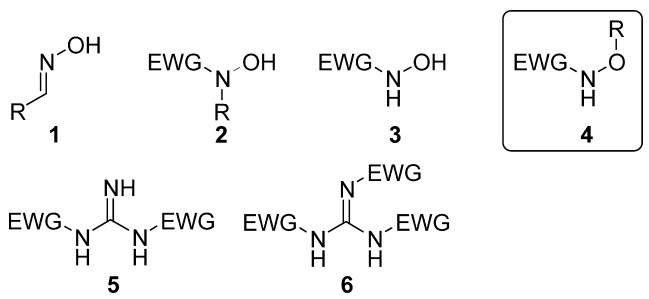

Fig. 1. Heteroatom Nucleophiles in Transition Metal-Catalyzed Allylic Substitution

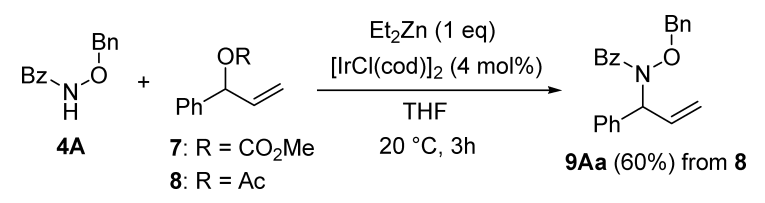

Chart 1. Iridium-Catalyzed Allylic Substitution of Hydroxylamine 4A

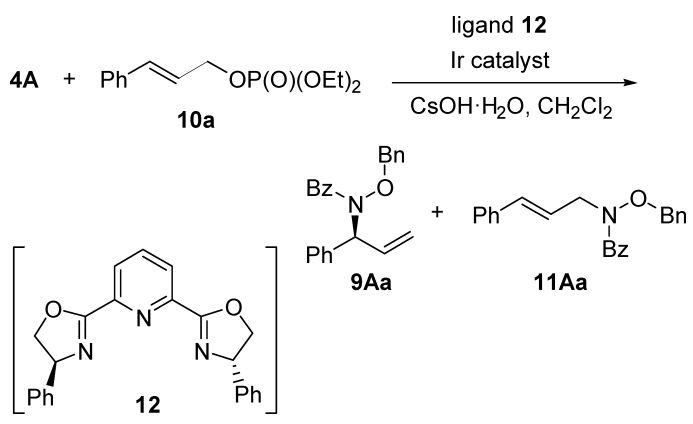

Chart 2. Regio- and Enantioselective Iridium-Catalyzed Allylic Substitution 
Table 1. Enantioselective Reaction of Hydroxylamine $\mathbf{4 A}$ with $\mathbf{1 0 a}{ }^{a}$

\begin{tabular}{|c|c|c|c|c|c|c|}
\hline Entry & Catalyst & $\mathrm{CsOH} \cdot \mathrm{H}_{2} \mathrm{O}$ & $\begin{array}{c}T \\
\left({ }^{\circ} \mathrm{C}\right)\end{array}$ & $\begin{array}{l}\text { Time } \\
\text { (h) }\end{array}$ & $\begin{array}{c}\left.\% \text { Yield }^{b}\right) \\
(\text { Ratio) })^{c)}\end{array}$ & $\begin{array}{c}\mathrm{Ee} \\
(\%)^{d)}\end{array}$ \\
\hline 1 & {$[\operatorname{IrCl}(\operatorname{cod})]_{2}$} & $1.0 \mathrm{eq}$ & +20 & 1 & $94(76: 24)$ & 79 \\
\hline 2 & {$[\mathrm{IrCl}(\operatorname{cod})]_{2}$} & $1.0 \mathrm{eq}$ & -20 & 8 & $89(86: 14)$ & 92 \\
\hline 3 & {$[\mathrm{IrCl}(\operatorname{cod})]_{2}$} & $1.0 \mathrm{eq}$ & -40 & 17 & $86(90: 10)$ & 92 \\
\hline 4 & {$[\mathrm{IrCl}(\operatorname{cod})]_{2}$} & $1.0 \mathrm{eq}$ & -60 & 40 & $45(87: 13)$ & 87 \\
\hline 5 & {$[\mathrm{IrCl}(\operatorname{cod})]_{2}$} & $0.5 \mathrm{eq}$ & -20 & 18 & $45(76: 24)$ & 79 \\
\hline 6 & {$[\operatorname{IrCl}(\operatorname{cod})]_{2}$} & none & -20 & 50 & NR & \\
\hline 7 & {$[\mathrm{IrOMe}(\operatorname{cod})]_{2}$} & $1.0 \mathrm{eq}$ & -20 & 3 & $94(86: 14)$ & 95 \\
\hline 8 & {$[\mathrm{IrOMe}(\operatorname{cod})]_{2}$} & none & -20 & 50 & NR & \\
\hline 9 & {$[\mathrm{IrCl}(\text { coe })]_{2}$} & $1.0 \mathrm{eq}$ & -20 & 50 & trace & \\
\hline
\end{tabular}

a) Reactions were carried out with $\mathbf{4 A}$ and $\mathbf{1 0 a}$ in $\mathrm{CH}_{2} \mathrm{Cl}_{2}$ in the presence of catalyst (4 mol\%) and pybox $\left.\mathbf{1 2}(8 \mathrm{~mol} \%) . \quad b\right)$ Combined yields. $\quad c$ ) Ratio for $\left.\mathbf{9 A a}: \mathbf{1 1} \mathrm{Aa} . \quad d\right)$ Enantioselectivities were determined by HPLC analysis.

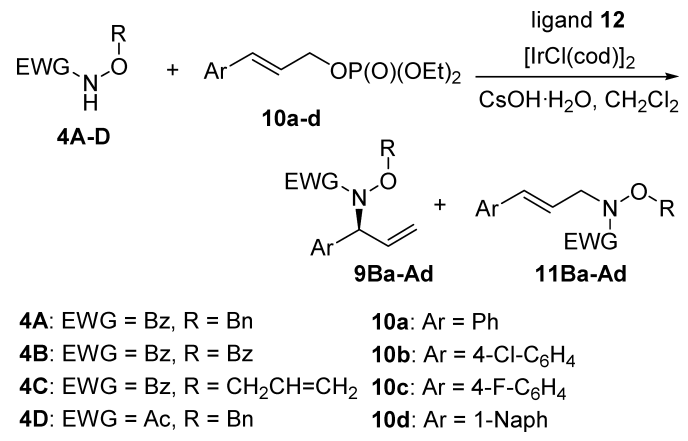

Chart 3. Regio- and Enantioselective Amination Using 4A-D

too, good yields of 9Aa were obtained with reasonable regioand enantioselectivities by employing $\mathrm{CsOH} \cdot \mathrm{H}_{2} \mathrm{O}$ or $\mathrm{Ba}(\mathrm{OH})_{2} \cdot \mathrm{H}_{2} \mathrm{O}$ as a base. The results using $\mathrm{Ba}(\mathrm{OH})_{2} \cdot \mathrm{H}_{2} \mathrm{O}$ have been described in our report ${ }^{19)}$; thus, Table 1 outlines the optimization of reaction conditions using $\mathrm{CsOH} \cdot \mathrm{H}_{2} \mathrm{O}$. To a solution of hydroxylamine $4 \mathrm{~A}$ and $\mathrm{CsOH} \cdot \mathrm{H}_{2} \mathrm{O}$ in $\mathrm{CH}_{2} \mathrm{Cl}_{2}$ was added a solution of the phosphate 10a, $[\mathrm{IrCl}(\operatorname{cod})]_{2}$ ( $4 \mathrm{~mol} \%$ ) and ligand $12(8 \mathrm{~mol} \%)$ in $\mathrm{CH}_{2} \mathrm{Cl}_{2}$, and then the reaction mixture was stirred at $20^{\circ} \mathrm{C}$ for $1 \mathrm{~h}$ (entry 1). The reaction proceeded smoothly to give the branched product 9Aa and the linear product 11 Aa in 94\% combined yield although low regioselectivity was observed. Enantiomeric excess of 9Aa was determined to be $79 \%$ by high performance liquid chromatography analysis using Chiralcel OD-H. The degree of regio- and enantioselectivities was shown to be dependent on the reaction temperature (entries 2-4). Thus, changing the temperature from 20 to $-20^{\circ} \mathrm{C}$ led to an increase in regioselectivity to $86: 14$ and enantioselectivity to $92 \%$ ee (entry 2). The branched product 9Aa was also obtained with $92 \%$ ee, after being stirred at $-40^{\circ} \mathrm{C}$ for $17 \mathrm{~h}$ (entry 3 ). In the absence of ligand 12, the iridium-catalyzed reaction of 4A with phosphate 10a did not occur. This result indicates the remarkable background reaction did not proceed under the present mild conditions using $\mathrm{CsOH} \cdot \mathrm{H}_{2} \mathrm{O}$. The reaction proceeded slowly at $-60^{\circ} \mathrm{C}$ to afford 9Aa with $87 \%$ ee in $87: 13$ ratio (entry 4 ). The reaction did not proceed effectively when 0.5 eq of $\mathrm{CsOH} \cdot \mathrm{H}_{2} \mathrm{O}$ was employed (entry 5). In the absence of $\mathrm{CsOH} \cdot \mathrm{H}_{2} \mathrm{O}$, practically no reaction occurred (entry 6). The use of $[\operatorname{IrOMe}(\operatorname{cod})]_{2}$ led to an increase in enantioselectivity to $95 \%$ ee (entry 7 ). However, in the absence of base, the reaction using [IrOMe (cod) $]_{2}$ did not proceed (entry 8$)$. In contrast to $[\mathrm{IrCl}(\operatorname{cod})]_{2}$ and $[\operatorname{IrOMe}(\operatorname{cod})]_{2}$,
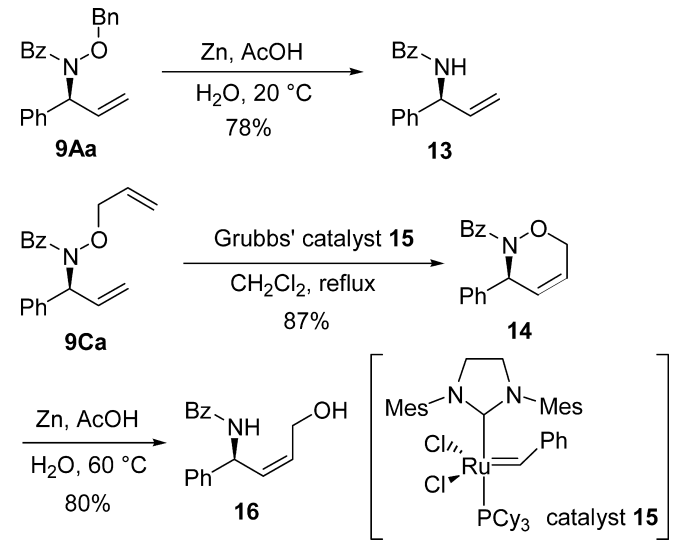

Chart 4. Conversion into Functionalized Compounds

the reaction did not proceed effectively when $[\operatorname{IrCl}(\mathrm{coe})]_{2}$ was employed (entry 9).

To gain further insight into the reactivity of hydroxylamines, several hydroxylamines $\mathbf{4 A}-\mathbf{D}$ and allylic substrates 10a-d were tested under the reaction conditions using $[\mathrm{IrCl}(\mathrm{cod})]_{2}$ (Chart 3). The nitrogen atom of hydroxylamine 4B having two electron-withdrawing substituents acted as a reactive nucleophile in allylic substitution (Table 2, entries 1 and 2). The reaction at $-20^{\circ} \mathrm{C}$ gave the branched product 9Ba with $87 \%$ ee in a $73: 23$ regioselectivity (entry 1). When the reaction was carried out at $-40^{\circ} \mathrm{C}$, the product 9Ba was obtained with $85 \%$ ee (entry 2 ). The hydroxylamine $4 \mathrm{C}$ having $\mathrm{N}$-benzoyl and $O$-allyl groups worked well as a nitrogen nucleophile (entries 3 and 4). Changing the temperature from -20 to $-40^{\circ} \mathrm{C}$ led to a decrease in regio- and enantioselectivity (entry 4). In contrast, moderate enantioselectivities were observed in the reaction with hydroxylamine 4D having $\mathrm{N}$-acetyl and $O$-benzyl groups (entries 5 and 6). Phosphates 10b-d worked well, allowing facile incorporation of structural variety (entries 7-11). The use of phosphate 10d having 1-naphthyl group led to an increase in regioselectivity to $>95: 5$ and enantioselectivity to $96 \%$ ee (entries 10 and 11 ).

The branched products can be converted into functionalized allylic compounds (Chart 4). The branched product 9Aa was easily converted into $\mathbf{1 3}$. The absolute configuration of product 13 was determined to be $S$ upon comparison with authentic compound $(R)-13{ }^{85)}$ The combination of intermolecular allylic substitution with metathesis is a useful method for the synthesis of heterocycles as demonstrated by Evans. ${ }^{25,26,86)}$ 
Table 2. Reaction of Hydroxylamines $\mathbf{4 A}-\mathbf{D}$ with $\mathbf{1 0 a}-\mathbf{d}^{a)}$

\begin{tabular}{|c|c|c|c|c|c|c|}
\hline Entry & $\begin{array}{l}\text { Hydroxyl- } \\
\text { amine }\end{array}$ & Phosphate & $\begin{array}{c}T \\
\left({ }^{\circ} \mathrm{C}\right)\end{array}$ & $\begin{array}{l}\text { Time } \\
\text { (h) }\end{array}$ & $\begin{array}{c}\left.\% \text { Yield }^{b}\right) \\
\quad(\text { ratio })\end{array}$ & $\begin{array}{c}\mathrm{Ee} \\
(\%)^{c)}\end{array}$ \\
\hline 1 & $4 B$ & $10 \mathbf{a}$ & -20 & 12 & $73(\mathbf{9 B a}: \mathbf{1 1 B a}=73: 27)$ & 87 \\
\hline 2 & $4 B$ & $10 \mathbf{a}$ & -40 & 12 & $44(\mathbf{9 B a}: \mathbf{1 1} \mathbf{B a}=72: 28)$ & 85 \\
\hline 3 & $4 \mathrm{C}$ & $10 \mathbf{a}$ & -20 & 8 & $88(\mathbf{9 C a}: \mathbf{1 1 C a}=89: 11)$ & 94 \\
\hline 4 & $4 \mathrm{C}$ & $10 \mathbf{a}$ & -40 & 50 & $47(\mathbf{9 C a}: \mathbf{1 1 C a}=87: 13)$ & 89 \\
\hline 5 & 4D & $10 \mathbf{a}$ & +20 & 12 & $84($ 9Da $:$ 11Da $=89: 11)$ & 33 \\
\hline 6 & 4D & $10 \mathbf{a}$ & -20 & 65 & $69(9 D a:$ 11Da $=90: 10)$ & 65 \\
\hline 7 & $4 \mathrm{~A}$ & $10 b$ & -20 & 20 & $75(\mathbf{9 A b}: \mathbf{1 1} \mathbf{A b}=70: 30)$ & 87 \\
\hline 8 & $4 \mathrm{~A}$ & $10 \mathrm{c}$ & -20 & 30 & $67(\mathbf{9 A c}: \mathbf{1 1 A c}=71: 29)$ & 83 \\
\hline 9 & $4 \mathrm{~A}$ & $10 \mathrm{c}$ & -40 & 65 & $45(\mathbf{9 A c}: \mathbf{1 1 A c}=80: 20)$ & 82 \\
\hline 10 & $4 \mathrm{~A}$ & $10 d$ & -20 & 30 & $95(\mathbf{9 A d}: \mathbf{1 1} \mathbf{A d}=>95: 5)$ & 96 \\
\hline 11 & $4 \mathrm{~A}$ & $10 d$ & -40 & 65 & $56(\mathbf{9 A d}: \mathbf{1 1} \mathbf{A d}=>95: 5)$ & 96 \\
\hline
\end{tabular}

a) Reactions were carried out with $\mathbf{4} \mathbf{A}-\mathbf{D}$ and $\mathbf{1 0 a}-\mathbf{d}$ in $\mathrm{CH}_{2} \mathrm{Cl}_{2}$ in the presence of $[\operatorname{IrCl}(\mathrm{cod})]_{2}(4 \mathrm{~mol} \%)$ and pybox $\mathbf{1 2}(8 \mathrm{~mol} \%)$. b) Combined yields. c) Enantioselectivities were determined by HPLC analysis.

Table 3. Reaction of Amines $\mathbf{1 7 A}-\mathbf{C}$ with $\mathbf{1 0 \mathbf { a } ^ { a }}$

\begin{tabular}{|c|c|c|c|c|c|c|}
\hline Entry & Amine & Base & $\begin{array}{c}T \\
\left({ }^{\circ} \mathrm{C}\right)\end{array}$ & $\begin{array}{l}\text { Time } \\
\text { (h) }\end{array}$ & $\begin{array}{c}\% \text { Yield }^{b)} \\
\left(\text { ratio }^{c)}\right.\end{array}$ & $\begin{array}{c}\mathrm{Ee} \\
(\%)^{d)}\end{array}$ \\
\hline 1 & 17A & $\mathrm{CsOH} \cdot \mathrm{H}_{2} \mathrm{O}$ & -20 & 1 & $91(71: 29)$ & 95 \\
\hline 2 & 17A & $\mathrm{CsOH} \cdot \mathrm{H}_{2}^{2} \mathrm{O}$ & -40 & 2 & $86(70: 30)$ & 87 \\
\hline 3 & 17A & none & -20 & 24 & $53(67: 33)$ & 76 \\
\hline 4 & 17B & $\mathrm{CsOH} \cdot \mathrm{H}_{2} \mathrm{O}$ & -20 & 20 & $66(78: 22)^{e)}$ & 94 \\
\hline 5 & $17 \mathrm{C}$ & $\mathrm{CsOH} \cdot \mathrm{H}_{2} \mathrm{O}$ & +20 & 5 & $87(71: 29)$ & 56 \\
\hline 6 & $17 \mathrm{C}$ & $\mathrm{CsOH} \cdot \mathrm{H}_{2} \mathrm{O}$ & -20 & 65 & $67(68: 32)$ & 71 \\
\hline
\end{tabular}

a) Reactions were carried out with $\mathbf{1 7 A}-\mathbf{C}$ and phosphate 10a in $\mathrm{CH}_{2} \mathrm{Cl}_{2}$ in the presence of $[\mathrm{IrCl}(\mathrm{cod})]_{2}(4 \mathrm{~mol} \%)$ and pybox $\mathbf{1 2}(8 \mathrm{~mol} \%)$. b) Combined yields. c) Ratio for 18A-C: 19A-C. d) Enantioselectivities were determined by HPLC analysis. e) A small amount of dicinnamylated product was obtained.

$$
\begin{aligned}
& \text { 17A-C } \\
& \text { 17A: } \mathrm{R}^{1}=\mathrm{Bn}, \mathrm{R}^{2}=\mathrm{Bn} \\
& \text { 17B: } \mathrm{R}^{1}=\mathrm{Bn}, \mathrm{R}^{2}=\mathrm{H} \\
& \text { 17C: } \mathrm{R}^{1}=\mathrm{Bn}, \mathrm{R}^{2}=\mathrm{OBn}
\end{aligned}
$$

Chart 5. Regio- and Enantioselective Amination Using Other Amines

Cyclic compound 14 was obtained by ring-closing metathesis (RCM) reaction of 9Ca using Grubbs' 2nd gen. catalyst $\mathbf{1 5}$. The cleavage of $\mathrm{N}-\mathrm{O}$ bond of $\mathbf{1 4}$ was achieved by reduction using $\mathrm{Zn}$ and $\mathrm{AcOH}$ to give the aminoalcohol 16 in $80 \%$ yield.

We next studied the asymmetric reaction with alkylamines as compared with hydroxylamines (Chart 5). Although $\mathrm{N}, \mathrm{N}$ dibenzylamine 17A has nucleophilic property even in the absence of a base, excellent enantioselectivity and chemical efficiency were obtained when $\mathrm{CsOH} \cdot \mathrm{H}_{2} \mathrm{O}$ was employed (Table 3, entries $1-3$ ). In the presence of $\mathrm{CsOH} \cdot \mathrm{H}_{2} \mathrm{O}$, the reaction proceeded smoothly at $-20{ }^{\circ} \mathrm{C}$ to give the branched product $\mathbf{1 8 A}$ with $95 \%$ ee within $1 \mathrm{~h}$ (entry 1 ). The reaction at $-40{ }^{\circ} \mathrm{C}$ afforded the product $\mathbf{1 8 \mathbf { A }}$ with $87 \%$ ee (entry 2 ). In the absence of $\mathrm{CsOH} \cdot \mathrm{H}_{2} \mathrm{O}$, the reaction with 17A was less effective (entry 3). Similar trend was observed in the reaction with $N$-benzylamine 17B. The product $\mathbf{1 8 B}$ was obtained with $94 \%$ ee under the reaction conditions using $\mathrm{CsOH} \cdot \mathrm{H}_{2} \mathrm{O}$ (entry 4). $\mathrm{N}, \mathrm{O}$-Dibenzylhydroxylamine $17 \mathrm{C}$ worked well (entries 5 and 6). Treatment of phosphate $10 \mathbf{a}$ with $17 \mathbf{C}$ at $-20^{\circ} \mathrm{C}$ gave the branched product $\mathbf{1 8 C}$ with $71 \%$ ee (entry $6)$.

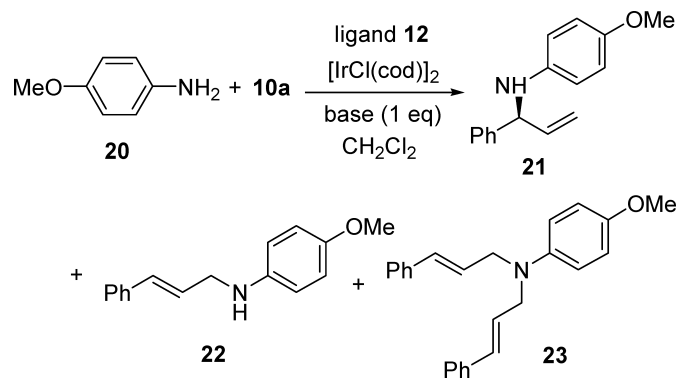

Chart 6. Regio- and Enantioselective Amination Using 20

Table 4. Reaction of $\mathbf{2 0}$ with $\mathbf{1 0} \mathbf{a}^{a)}$

\begin{tabular}{cccccc}
\hline \hline Entry & Base & $\begin{array}{c}T \\
\left({ }^{\circ} \mathrm{C}\right)\end{array}$ & $\begin{array}{c}\text { Time } \\
(\mathrm{h})\end{array}$ & $\begin{array}{c}\% \text { Yield } \\
(\text { ratio })^{c}\end{array}$ & $\begin{array}{c}\text { Ee } \\
\left.(\%)^{d}\right)\end{array}$ \\
\hline 1 & $\mathrm{CsOH} \cdot \mathrm{H}_{2} \mathrm{O}$ & -20 & 3 & $95(81: 9: 10)$ & 88 \\
2 & $\mathrm{CsOH} \cdot \mathrm{H}_{2} \mathrm{O}$ & -40 & 24 & $86(80: 10: 10)$ & 87 \\
3 & none & -40 & 24 & $55(71: 15: 14)$ & 72 \\
\hline
\end{tabular}

a) Reactions were carried out with 20 and phospate $\mathbf{1 0 a}$ in $\mathrm{CH}_{2} \mathrm{Cl}_{2}$ in the presence of $[\mathrm{IrCl}(\mathrm{cod})]_{2}(4 \mathrm{~mol} \%)$ and pybox $12(8 \mathrm{~mol} \%)$. b) Combined yields. c) Ratio for $21: 22: 22$. d) Enantioselectivities were determined by HPLC analysis.

Next, the asymmetric reaction with less reactive aniline derivative was investigated (Chart 6). Although $p$-anisidine 20 participated in the present reaction, formation of branched product 21, linear product $\mathbf{2 2}$ and diallylated product $\mathbf{2 3}$ were observed. The use of $\mathrm{CsOH} \cdot \mathrm{H}_{2} \mathrm{O}$ as a base led to an increase in regioselectivity and enantioselectivity (Table 4). p-Anisidine 20 worked well at $-20^{\circ} \mathrm{C}$ to give the branched product 21 with $88 \%$ ee in $81: 9: 10$ ratio (entry 1 ). The product 21 was also obtained with $87 \%$ ee after being stirred at 
Table 5. Reaction of $\mathbf{2 4}$ with $\mathbf{1 0 \mathbf { a } ^ { a }}$

\begin{tabular}{clrrrr}
\hline \hline Entry & \multicolumn{1}{c}{ Base } & $\begin{array}{c}T \\
\left({ }^{\circ} \mathrm{C}\right)\end{array}$ & $\begin{array}{c}\text { Time } \\
(\mathrm{h})\end{array}$ & $\begin{array}{c}\% \mathrm{Yield}^{b)} \\
(\text { ratio })^{c)}\end{array}$ & $\begin{array}{c}\text { ee } \\
\left.(\%)^{d}\right)\end{array}$ \\
\hline 1 & $\mathrm{CsOH} \cdot \mathrm{H}_{2} \mathrm{O}$ & +20 & 48 & $61(78: 22)$ & 24 \\
2 & $\mathrm{Ba}(\mathrm{OH})_{2} \cdot \mathrm{H}_{2} \mathrm{O}$ & +20 & 1 & $84(87: 13)$ & 45 \\
3 & $\mathrm{Ba}(\mathrm{OH})_{2} \cdot \mathrm{H}_{2} \mathrm{O}$ & -20 & 48 & $65(98: 2)$ & 51 \\
4 & $\mathrm{~K}_{2} \mathrm{CO}_{3}$ & +20 & 3 & $86(83: 17)$ & 58 \\
5 & $\mathrm{~K}_{2} \mathrm{CO}_{3}$ & -20 & 48 & $88(92: 8)$ & 72 \\
\hline
\end{tabular}

a) Reactions were carried out with $\mathbf{2 4}$ and phosphate 10a in $\mathrm{CH}_{2} \mathrm{Cl}_{2}$ in the presence of $[\mathrm{IrCl}(\operatorname{cod})]_{2}(4 \mathrm{~mol} \%)$ and pybox $12(8 \mathrm{~mol} \%)$. b) Combined yields. c) Ratio for $25: 26$. d) Enantioselectivities were determined by HPLC analysis.

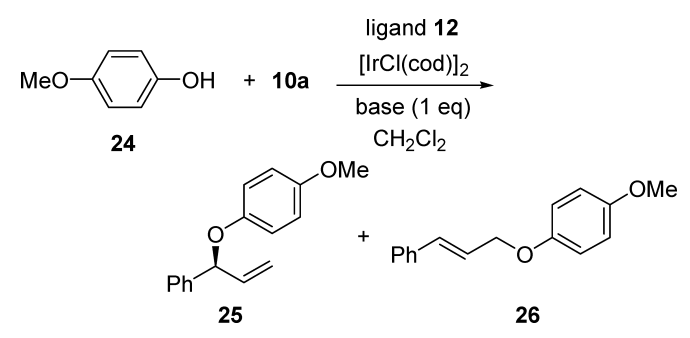

Chart 7. Regio- and Enantioselective Amination Using 24

$-40^{\circ} \mathrm{C}$ for $24 \mathrm{~h}$ (entry 2). In the absence of $\mathrm{CsOH} \cdot \mathrm{H}_{2} \mathrm{O}$, treatment of phosphate 10a with 20 gave 21 with $72 \%$ ee (entry 3). Although the effect of base on these enantioselectivities was questioned, we assume that one role would involve the activation of catalyst.

We finally investigated the asymmetric reaction with 4methoxyphenol 24 (Chart 7). In the presence of $\mathrm{CsOH} \cdot \mathrm{H}_{2} \mathrm{O}$, the reaction of $\mathbf{1 0 a}$ with 4-methoxyphenol $\mathbf{2 4}$ proceeded slowly to afford the branched product $\mathbf{2 5}$ with poor enantioselectivity (Table 5, entry 1 ). To improve the reactivity and selectivities, the effect of bases was next studied (entries 2 5). Although $\mathrm{Ba}(\mathrm{OH})_{2} \cdot \mathrm{H}_{2} \mathrm{O}$ was less effective for the present reaction, improved selectivities and chemical efficiencies were observed when $\mathrm{K}_{2} \mathrm{CO}_{3}$ was employed. The reaction using $\mathrm{K}_{2} \mathrm{CO}_{3}$ at $-20{ }^{\circ} \mathrm{C}$ gave the $72 \%$ ee of the branched product 25 with a $92: 8$ regioselectivity (entry 5). Results from this study show that the iridium complex of pybox ligand is able to catalyze the allylic substitution with less reactive $O$-nucleophiles such as phenols.

In conclusion, we have demonstrated that the iridiumcomplex of pybox catalyzed the allylic substitution with hydroxylamines in good enantioselectivities. Good regio- and enantioselectivities were also achieved in allylic substitution with alkylamines, $p$-anisidine, and 4-methoxyphenol.

\section{Experimental}

General Melting points are uncorrected. ${ }^{1} \mathrm{H}$ - and ${ }^{13} \mathrm{C}-\mathrm{NMR}$ spectra were recorded at $500 \mathrm{MHz}$, and at $125 \mathrm{MHz}$, respectively. IR spectra were recorded using Fourier transform (FT)-IR apparatus. Mass spectra were obtained by EI or FAB methods. Preparative TLC separations were carried out on precoated silica gel plates $\left(\mathrm{E}\right.$. Merck $\left.60 \mathrm{~F}_{254}\right)$. Flash column chromatography was performed using E. Merck Kieselgel 60 (230 - 400 mesh). $[\alpha]_{D}$ values are measured in $10^{-1} \mathrm{deg} \mathrm{cm}^{2} \mathrm{~g}^{-1}$. The ratios of products were determined by ${ }^{1} \mathrm{H}-\mathrm{NMR}$ analysis. Enantiomeric excess was determined by high performance liquid chromatography (HPLC) analysis. Products 19A, ${ }^{87)}$ 19B $,{ }^{88)} \mathbf{2 2},{ }^{89)} \mathbf{2 3},{ }^{89)}$ and $\mathbf{2 6}{ }^{90)}$ are the known compounds.

General Procedure for Enantioselective Allylic Substitution of Amines A mixture of amine $4 \mathbf{A}-\mathbf{D}$ or $\mathbf{1 7 A}-\mathbf{C}(1.0 \mathrm{mmol})$ and $\mathrm{CsOH}$ $\mathrm{H}_{2} \mathrm{O}(168 \mathrm{mg}, 1.0 \mathrm{mmol})$ in $\mathrm{CH}_{2} \mathrm{Cl}_{2}(4.0 \mathrm{ml})$ was stirred under argon atmosphere at $20^{\circ} \mathrm{C}$ for $10 \mathrm{~min}$. To the reaction mixture was added a solution of allylic phosphate 10a-d (1.5 mmol), pybox $12(29.6 \mathrm{mg}, 0.080 \mathrm{mmol})$ and
[ $\mathrm{IrCl}(\mathrm{cod})]_{2}(26.9 \mathrm{mg}, 0.040 \mathrm{mmol})$ in $\mathrm{CH}_{2} \mathrm{Cl}_{2}(2.0 \mathrm{ml})$ at temperature indicated in Tables 2-4. After the reaction was completed, the reaction mixture was diluted with saturated $\mathrm{NH}_{4} \mathrm{Cl}$ and then extracted with AcOEt. The organic phase was dried over $\mathrm{MgSO}_{4}$ and concentrated at reduced pressure. The ratio of products was determined by ${ }^{1} \mathrm{H}-\mathrm{NMR}$ analysis of crude products. Purification of the residue by preparative TLC (hexane: AcOEt= $5: 1-25: 1,2$-fold development) afforded the products $9 \mathbf{A a}-\mathbf{A d}, \mathbf{1 1 A a}-$ Ad, $18 \mathrm{~A}-\mathrm{C}$, and $19 \mathrm{~A}-\mathrm{C}$.

$\boldsymbol{N}$-(Benzyloxy)- $\boldsymbol{N}$-((S)-1-phenylallyl)benzamide (9Aa) As a colorless oil: IR $\left(\mathrm{CHCl}_{3}\right) \mathrm{cm}^{-1}: 1635,1495,1451 .{ }^{1} \mathrm{H}-\mathrm{NMR}\left(\mathrm{CDCl}_{3}\right) \delta: 7.70(2 \mathrm{H}, \mathrm{d}$, $J=7.0 \mathrm{~Hz}), 7.60-7.15(11 \mathrm{H}, \mathrm{m}), 6.83(2 \mathrm{H}, \mathrm{d}, J=7.0 \mathrm{~Hz}), 6.32(1 \mathrm{H}$, ddd, $J=17.7,11.0,6.1 \mathrm{~Hz}), 6.15(1 \mathrm{H}, \mathrm{d}, J=6.1 \mathrm{~Hz}), 5.39(1 \mathrm{H}, \mathrm{d}, J=17.7 \mathrm{~Hz})$, $5.38(1 \mathrm{H}, \mathrm{d}, J=11.0 \mathrm{~Hz}), 4.48(1 \mathrm{H}, \mathrm{d}, J=8.5 \mathrm{~Hz}), 4.11(1 \mathrm{H}, \mathrm{d}, J=8.5 \mathrm{~Hz})$. ${ }^{13} \mathrm{C}-\mathrm{NMR}\left(\mathrm{CDCl}_{3}\right) \delta: 170.6,138.1,134.9,134.2,134.1,130.6,129.5,128.8$, 128.6 (2C), 128.3, 128.3, 128.1, 118.6, 78.5, 64.1. One carbon peak was missing due to overlapping. MS (EI $\left.{ }^{+}\right) m / z: 343\left(\mathrm{M}^{+}, 11\right), 115$ (100). HR-MS $m / z: 343.1570$ (Calcd for $\mathrm{C}_{23} \mathrm{H}_{21} \mathrm{NO}_{2}: 343.1572$ ). HPLC (Chiralcel OD-H, hexane/2-propanol $=95 / 5,0.5 \mathrm{ml} / \mathrm{min}, 254 \mathrm{~nm}) t_{\mathrm{R}}(S)=15.8 \mathrm{~min}, t_{\mathrm{R}}(R)=18.1$ min. A sample of $92 \%$ ee $(S)$ by HPLC analysis gave $[\alpha]_{\mathrm{D}}^{29}-33.8(c=1.02$, $\left.\mathrm{CHCl}_{3}\right)$.

$\boldsymbol{N}$-(Benzoyloxy)- $\boldsymbol{N}$-((S)-1-phenylallyl)benzamide (9Ba) As a colorless oil: IR $\left(\mathrm{CHCl}_{3}\right) \mathrm{cm}^{-1}: 1767,1665,1495,1451 .{ }^{1} \mathrm{H}-\mathrm{NMR}\left(\mathrm{CDCl}_{3}\right) \delta: 7.84$ $(2 \mathrm{H}, \mathrm{d}, J=7.6 \mathrm{~Hz}), 7.68(2 \mathrm{H}, \mathrm{d}, J=7.3 \mathrm{~Hz}), 7.55(1 \mathrm{H}, \mathrm{t}, J=7.6 \mathrm{~Hz}), 7.45$ $7.22(10 \mathrm{H}, \mathrm{m}), 6.22(1 \mathrm{H}, \mathrm{ddd}, J=17.1,8.9,6.1 \mathrm{~Hz}), 6.11(1 \mathrm{H}, \mathrm{d}, J=6.1 \mathrm{~Hz})$, $5.40(1 \mathrm{H}, \mathrm{d}, J=8.9 \mathrm{~Hz}), 5.37(1 \mathrm{H}, \mathrm{d}, J=17.1 \mathrm{~Hz}) \cdot{ }^{13} \mathrm{C}-\mathrm{NMR}\left(\mathrm{CDCl}_{3}\right) \delta$ : $170.6,164.0,134.0,133.9,133.8,131.1,129.8,128.6,128.4,128.1,127.8$, $127.2,119.4,66.1$. Two carbon peaks were missing due to overlapping. MS $\left(\mathrm{EI}^{+}\right) \mathrm{m} / \mathrm{z}: 357\left(\mathrm{M}^{+}, 7\right), 116$ (100). HR-MS $\mathrm{m} / \mathrm{z}: 357.1371$ (Calcd for $\mathrm{C}_{23} \mathrm{H}_{19} \mathrm{NO}_{3}$ : 357.1365). HPLC (Chiralcel AD-H, hexane/2-propanol=90/10, $1.0 \mathrm{ml} / \mathrm{min}, 254 \mathrm{~nm}) t_{\mathrm{R}}(S)=19.7 \mathrm{~min}, t_{\mathrm{R}}(R)=15.1 \mathrm{~min}$. A sample of $87 \%$ ee $(S)$ by HPLC analysis gave $[\alpha]_{\mathrm{D}}^{31}+2.6\left(c=0.96, \mathrm{CHCl}_{3}\right)$.

$\boldsymbol{N}$-(Allyloxy)- $\boldsymbol{N}$-((S)-1-phenylallyl)benzamide (9Ca) As a colorless oil: IR $\left(\mathrm{CHCl}_{3}\right) \mathrm{cm}^{-1}: 1767,1665,1495,1451 .{ }^{1} \mathrm{H}-\mathrm{NMR}\left(\mathrm{CDCl}_{3}\right) \delta: 7.68$ $(2 \mathrm{H}, \mathrm{d}, J=7.0 \mathrm{~Hz}), 7.46-7.30(8 \mathrm{H}, \mathrm{m}), 6.30(1 \mathrm{H}, \mathrm{ddd}, J=17.2,10.4$, $6.4 \mathrm{~Hz}), 6.03(1 \mathrm{H}, \mathrm{d}, J=6.4 \mathrm{~Hz}), 5.51(1 \mathrm{H}, \mathrm{m}), 5.39(1 \mathrm{H}, \mathrm{d}, J=10.4 \mathrm{~Hz}), 5.38$ $(1 \mathrm{H}, \mathrm{d}, J=17.2 \mathrm{~Hz}), 5.06(1 \mathrm{H}, \mathrm{d}, J=10.4 \mathrm{~Hz}), 4.98(1 \mathrm{H}, \mathrm{d}, J=17.2 \mathrm{~Hz}), 4.03$ $(1 \mathrm{H}, \mathrm{m}), 3.75(1 \mathrm{H}, \mathrm{m}) .{ }^{13} \mathrm{C}-\mathrm{NMR}\left(\mathrm{CDCl}_{3}\right) \delta: 170.5,138.1,134.8,134.3$, 131.2, 130.6, 128.6, 128.5, 128.1, 128.0 (2C), 119.9, 118.6, 77.6, 64.4. MS $\left(\mathrm{EI}^{+}\right) \mathrm{m} / z: 293\left(\mathrm{M}^{+}, 3\right), 77$ (100). HR-MS m/z: 293.1412 (Calcd for $\left.\mathrm{C}_{19} \mathrm{H}_{19} \mathrm{NO}_{2}: 293.1416\right)$. HPLC (Chiralcel AD-H, hexane/2-propanol=90/10, $0.5 \mathrm{ml} / \mathrm{min}, 254 \mathrm{~nm}) t_{\mathrm{R}}(S)=19.2 \mathrm{~min}, t_{\mathrm{R}}(R)=14.2 \mathrm{~min}$. A sample of $94 \%$ ee $(S)$ by HPLC analysis gave $[\alpha]_{\mathrm{D}}^{25}+50.1\left(c=1.3, \mathrm{CHCl}_{3}\right)$.

$\boldsymbol{N}$-(Benzoyloxy)- $\boldsymbol{N}$-((S)-1-phenylallyl)acetamide (9Da) As a colorless oil: IR $\left(\mathrm{CHCl}_{3}\right) \mathrm{cm}^{-1}: 1767,1665,1495,1451 .{ }^{1} \mathrm{H}-\mathrm{NMR}\left(\mathrm{CDCl}_{3}\right) \delta: 7.46$ $(2 \mathrm{H}, \mathrm{d}, J=7.0 \mathrm{~Hz}), 7.42-7.30(6 \mathrm{H}, \mathrm{m}), 7.19-7.13(2 \mathrm{H}, \mathrm{m}), 6.26(1 \mathrm{H}$, ddd, $J=17.1,10.4,5.5 \mathrm{~Hz}), 6.12(1 \mathrm{H}, \mathrm{d}, J=5.5 \mathrm{~Hz}), 5.35(1 \mathrm{H}, \mathrm{d}, J=10.4 \mathrm{~Hz})$, $5.31(1 \mathrm{H}, \mathrm{d}, J=17.1 \mathrm{~Hz}), 4.67(1 \mathrm{H}, \mathrm{d}, J=9.8 \mathrm{~Hz}), 4.27(1 \mathrm{H}, \mathrm{d}, J=9.8 \mathrm{~Hz})$, $2.22(3 \mathrm{H}, \mathrm{s}) .{ }^{13} \mathrm{C}-\mathrm{NMR}\left(\mathrm{CDCl}_{3}\right) \delta: 173.6,138.2,134.4,134.0,129.0,128.8$ (2C), 128.5 (2C), 128.0, 118.5, 78.7, 63.2, 21.0. MS (EI $\left.{ }^{+}\right) m / z: 281\left(\mathrm{M}^{+}\right.$, 15), 91 (100). HR-MS m/z: 281.1410 (Calcd for $\mathrm{C}_{18} \mathrm{H}_{19} \mathrm{NO}_{2}: 281.1416$ ). HPLC (Chiralcel OD-H, hexane/2-propanol=95/5, $0.5 \mathrm{ml} / \mathrm{min}, 254 \mathrm{~nm}$ ) $t_{\mathrm{R}}$ $(S)=18.9 \mathrm{~min}, t_{\mathrm{R}}(R)=20.5 \mathrm{~min}$. A sample of $65 \%$ ee $(S)$ by HPLC analysis gave $[\alpha]_{\mathrm{D}}^{29}-13.1\left(c=1.03, \mathrm{CHCl}_{3}\right)$.

$\boldsymbol{N}$-(Benzyloxy)- $\boldsymbol{N}$-((S)-1-(4-chlorophenyl)allyl)benzamide (9Ab) As a colorless oil: IR $\left(\mathrm{CHCl}_{3}\right) \mathrm{cm}^{-1}: 1639,1492,1449 .{ }^{1} \mathrm{H}-\mathrm{NMR}\left(\mathrm{CDCl}_{3}\right) \delta: 7.67$ $(2 \mathrm{H}, \mathrm{d}, J=7.0 \mathrm{~Hz}), 7.52-7.19(10 \mathrm{H}, \mathrm{m}), 6.87(2 \mathrm{H}, \mathrm{d}, J=7.3 \mathrm{~Hz}), 6.29(1 \mathrm{H}$, ddd, $J=15.9,9.5,6.7 \mathrm{~Hz}), 6.09(1 \mathrm{H}, \mathrm{d}, J=6.7 \mathrm{~Hz}), 5.40(1 \mathrm{H}, \mathrm{d}, J=9.5 \mathrm{~Hz})$, $5.37(1 \mathrm{H}, \mathrm{d}, J=15.9 \mathrm{~Hz}), 4.54(1 \mathrm{H}$, brd, $J=8.9 \mathrm{~Hz}), 4.22(1 \mathrm{H}$, brd, $J=8.9 \mathrm{~Hz}) .{ }^{13} \mathrm{C}-\mathrm{NMR}\left(\mathrm{CDCl}_{3}\right) \delta: 170.7,136.8,134.7,133.9$ (2C), 130.7, $130.0,129.4,128.8,128.4,128.2,128.1,119.2,78.7,63.7$. Two carbon peaks were missing due to overlapping. $\mathrm{MS}\left(\mathrm{EI}^{+}\right) \mathrm{m} / z: 377\left(\mathrm{M}^{+}, 2\right), 91$ (100). HR-MS m/z: 377.1187 (Calcd for $\mathrm{C}_{23} \mathrm{H}_{20} \mathrm{ClNO}_{2}: 377.1188$ ). HPLC (Chiralcel AD-H, hexane/2-propanol $=90 / 10,0.5 \mathrm{ml} / \mathrm{min}, 254 \mathrm{~nm}$ ) $t_{\mathrm{R}}$ $(S)=28.0 \mathrm{~min}, t_{\mathrm{R}}(R)=21.9 \mathrm{~min}$. A sample of $87 \%$ ee $(S)$ by HPLC analysis gave $[\alpha]_{\mathrm{D}}^{26}-3.7\left(c=1.15, \mathrm{CHCl}_{3}\right)$.

$N$-(Benzyloxy)- $N$-((S)-1-(4-fluorophenyl)allyl)benzamide (9Ac) As a colorless oil: IR $\left(\mathrm{CHCl}_{3}\right) \mathrm{cm}^{-1}: 1637,1510,1449 .{ }^{1} \mathrm{H}-\mathrm{NMR}\left(\mathrm{CDCl}_{3}\right) \delta: 7.68$ $(2 \mathrm{H}, \mathrm{d}, J=7.0 \mathrm{~Hz}), 7.52-7.38(5 \mathrm{H}, \mathrm{m}), 7.30-7.20(3 \mathrm{H}, \mathrm{m}), 7.07(2 \mathrm{H}, \mathrm{t}$, $J=8.5 \mathrm{~Hz}), 6.86(2 \mathrm{H}, \mathrm{d}, J=7.3 \mathrm{~Hz}), 6.29(1 \mathrm{H}, \mathrm{ddd}, J=17.1,10.4,6.4 \mathrm{~Hz})$, $6.11(1 \mathrm{H}, \mathrm{d}, J=6.4 \mathrm{~Hz}), 5.39(1 \mathrm{H}, \mathrm{d}, J=10.4 \mathrm{~Hz}), 5.37(1 \mathrm{H}, \mathrm{d}, J=17.1 \mathrm{~Hz})$, $4.52(1 \mathrm{H}$, brd, $J=8.9 \mathrm{~Hz}), 4.16(1 \mathrm{H}$, br d, $J=8.9 \mathrm{~Hz}) .{ }^{13} \mathrm{C}-\mathrm{NMR}\left(\mathrm{CDCl}_{3}\right) \delta$ : $170.7,162.6(\mathrm{~d}, J=247 \mathrm{~Hz}), 134.8,134.2,134.0,130.7,130.5,130.4,129.4$, $128.7,128.3,128.2,128.1,118.9,115.5$ (d, $J=21 \mathrm{~Hz}), 78.6,63.5$. Two carbon peaks were missing due to overlapping. $\mathrm{MS}\left(\mathrm{EI}^{+}\right) \mathrm{m} / z: 361\left(\mathrm{M}^{+}, 3\right), 91$ 
(100). HR-MS m/z: 361.1476 (Calcd for $\mathrm{C}_{23} \mathrm{H}_{20} \mathrm{FNO}_{2}:$ 361.1478). HPLC (Chiralcel AD-H, hexane $/ 2$-propanol $=90 / 10,0.5 \mathrm{ml} / \mathrm{min}, 254 \mathrm{~nm}) t_{\mathrm{R}}(S)=$ $23.9 \mathrm{~min}, t_{\mathrm{R}}(R)=20.6 \mathrm{~min}$. A sample of $83 \%$ ee $(S)$ by HPLC analysis gave $[\alpha]_{\mathrm{D}}^{26}-22.7\left(c=1.30, \mathrm{CHCl}_{3}\right)$.

$N$-(Benzyloxy)- $N$-((S)-1-(naphthalen-1-yl)allyl)benzamide (9Ad) As a colorless oil: IR $\left(\mathrm{CHCl}_{3}\right) \mathrm{cm}^{-1}: 1632,1511,1495,1449 .{ }^{1} \mathrm{H}-\mathrm{NMR}$ $\left(\mathrm{CDCl}_{3}\right) \delta: 8.37(1 \mathrm{H}, \mathrm{d}, J=8.5 \mathrm{~Hz}), 7.90(2 \mathrm{H}, \mathrm{d}, J=8.2 \mathrm{~Hz}), 7.76(3 \mathrm{H}, \mathrm{d}$, $J=7.9 \mathrm{~Hz}), 7.65(1 \mathrm{H}, \mathrm{t}, J=7.0 \mathrm{~Hz}), 7.57-7.35(5 \mathrm{H}, \mathrm{m}), 7.20-7.05(4 \mathrm{H}$, m), $6.50-6.40(3 \mathrm{H}, \mathrm{m}), 5.59(1 \mathrm{H}, \mathrm{d}, J=17.1 \mathrm{~Hz}), 5.48(1 \mathrm{H}, \mathrm{d}, J=10.7 \mathrm{~Hz})$, $3.94(1 \mathrm{H}, \mathrm{brs}), 3.36(1 \mathrm{H}, \mathrm{brs}) .{ }^{13} \mathrm{C}-\mathrm{NMR}\left(\mathrm{CDCl}_{3}\right) \delta: 170.1,134.8,134.1$, 133.7, 133.6, 133.4, 132.0, 130.5, 129.5, 129.3, 128.9, 128.5, 128.3, 128.1, 128.0, 127.8, 127.1, 126.1, 125.1, 123.5, 117.8, 78.4, 58.7. MS $\left(\mathrm{EI}^{+}\right) \mathrm{m} / \mathrm{z}$ : $393\left(\mathrm{M}^{+}, 6\right), 167$ (100). HR-MS m/z: 393.1727 (Calcd for $\mathrm{C}_{27} \mathrm{H}_{23} \mathrm{NO}_{2}$ : 393.1729). HPLC (Chiralcel AD-H, hexane $/ 2$-propanol $=90 / 10,0.5 \mathrm{ml} / \mathrm{min}$, $254 \mathrm{~nm}) t_{\mathrm{R}}(S)=17.4 \mathrm{~min}, t_{\mathrm{R}}(R)=14.9 \mathrm{~min}$. A sample of $96 \%$ ee $(S)$ by HPLC analysis gave $[\alpha]_{\mathrm{D}}^{28}-20.6\left(c=1.07, \mathrm{CHCl}_{3}\right)$.

$\mathrm{N}$-(Benzyloxy)- $\mathrm{N}$-cinnamylbenzamide (11Aa) As a colorless oil: IR $\left(\mathrm{CHCl}_{3}\right) \mathrm{cm}^{-1}: 1635,1496,1450 .{ }^{1} \mathrm{H}-\mathrm{NMR}\left(\mathrm{CDCl}_{3}\right) \delta: 7.67(2 \mathrm{H}, \mathrm{d}, J=$ $7.3 \mathrm{~Hz}), 7.49-7.22(11 \mathrm{H}, \mathrm{m}), 7.07(2 \mathrm{H}, \mathrm{d}, J=6.7 \mathrm{~Hz}), 6.61(1 \mathrm{H}, \mathrm{d}, J=$ $15.9 \mathrm{~Hz}), 6.31(1 \mathrm{H}, \mathrm{dt}, J=15.9,6.1 \mathrm{~Hz}), 4.72(2 \mathrm{H}, \mathrm{s}), 4.49(2 \mathrm{H}, \mathrm{d}$, $J=6.1 \mathrm{~Hz}) .{ }^{13} \mathrm{C}-\mathrm{NMR}\left(\mathrm{CDCl}_{3}\right) \delta: 170.3,136.5,134.5,134.3,134.0,130.6$, $129.5,128.8,128.6,128.5,128.3,128.1,127.9,126.6,123.4,77.1,50.5$. One carbon peak was missing due to overlapping. MS $\left(\mathrm{FAB}^{+}\right) \mathrm{m} / \mathrm{z}: 344$ $\left(\mathrm{M}+\mathrm{H}^{+}, 64\right), 117$ (100). HR-MS m/z: 344.1656 (Calcd for $\mathrm{C}_{23} \mathrm{H}_{22} \mathrm{NO}_{2}$ : 344.1651).

$N$-(Benzoyloxy)- $N$-cinnamylbenzamide (11Ba) As a colorless oil: IR $\left(\mathrm{CHCl}_{3}\right) \mathrm{cm}^{-1}: 1764,1663,1495,1449 .{ }^{1} \mathrm{H}-\mathrm{NMR}\left(\mathrm{CDCl}_{3}\right) \delta: 7.91(2 \mathrm{H}, \mathrm{d}$, $J=7.6 \mathrm{~Hz}), 7.65(2 \mathrm{H}, \mathrm{d}, J=7.0 \mathrm{~Hz}), 7.56(1 \mathrm{H}, \mathrm{d}, J=7.3 \mathrm{~Hz}), 7.44-7.21$ $(10 \mathrm{H}, \mathrm{m}), 6.61(1 \mathrm{H}, \mathrm{d}, J=15.9 \mathrm{~Hz}), 6.31(1 \mathrm{H}, \mathrm{dt}, J=15.9,6.4 \mathrm{~Hz}), 4.67(2 \mathrm{H}$, d, $J=6.4 \mathrm{~Hz}) .{ }^{13} \mathrm{C}-\mathrm{NMR}\left(\mathrm{CDCl}_{3}\right) \delta: 170.8,164.6,136.3,134.4,134.1$, 133.5, 131.1, 129.9, 128.7, 128.6, 128.3, 128.0, 127.9, 127.0, 126.6, 122.6, 52.3. $\mathrm{MS}_{\left(\mathrm{EI}^{+}\right)} \mathrm{m} / z$ : $357\left(\mathrm{M}^{+}, 3\right), 116$ (100). HR-MS m/z: 357.1369 (Calcd for $\left.\mathrm{C}_{23} \mathrm{H}_{19} \mathrm{NO}_{3}: 357.1365\right)$.

$N$-(Allyloxy)- $N$-cinnamylbenzamide (11Ca) As a colorless oil: IR $\left(\mathrm{CHCl}_{3}\right) \mathrm{cm}^{-1}: 1634,1495,1449,1427 .{ }^{1} \mathrm{H}-\mathrm{NMR}\left(\mathrm{CDCl}_{3}\right) \delta: 7.70(2 \mathrm{H}, \mathrm{d}$, $J=7.0 \mathrm{~Hz}), 7.46-7.23(8 \mathrm{H}, \mathrm{m}), 6.63(1 \mathrm{H}, \mathrm{d}, J=15.9 \mathrm{~Hz}), 6.34(1 \mathrm{H}, \mathrm{dt}$, $J=15.9,6.4 \mathrm{~Hz}), 5.71(1 \mathrm{H}, \mathrm{m}), 5.19(1 \mathrm{H}, \mathrm{d}, J=16.2 \mathrm{~Hz}), 5.18(1 \mathrm{H}, \mathrm{d}$, $J=11.0 \mathrm{~Hz}), 4.50(2 \mathrm{H}, \mathrm{d}, J=6.4 \mathrm{~Hz}), 4.26(2 \mathrm{H}, \mathrm{d}, J=6.4 \mathrm{~Hz}) .{ }^{13} \mathrm{C}-\mathrm{NMR}$ $\left(\mathrm{CDCl}_{3}\right) \delta: 170.1,136.5,134.4,133.8,131.5,130.6,128.6,128.3,128.0$ 127.9, 126.5, 123.4, 120.5, 76.0, 50.6. $\mathrm{MS}\left(\mathrm{EI}^{+}\right) \mathrm{m} / \mathrm{z}: 293\left(\mathrm{M}^{+}, 2\right), 77(100)$. HR-MS $m / z: 293.1419$ (Calcd for $\mathrm{C}_{19} \mathrm{H}_{19} \mathrm{NO}_{2}: 293.1416$ ).

$N$-(Benzyloxy)- $N$-cinnamylacetamide (11Da) As a colorless oil: IR $\left(\mathrm{CHCl}_{3}\right) \mathrm{cm}^{-1}: 1655,1496,1434 .{ }^{1} \mathrm{H}-\mathrm{NMR}\left(\mathrm{CDCl}_{3}\right) \delta: 7.40-7.21(10 \mathrm{H}$, $\mathrm{m}), 6.55(1 \mathrm{H}, \mathrm{d}, J=15.9 \mathrm{~Hz}), 6.25(1 \mathrm{H}, \mathrm{dt}, J=15.9,5.8 \mathrm{~Hz}), 4.87(2 \mathrm{H}, \mathrm{s})$, $4.39(2 \mathrm{H}, \mathrm{d}, J=5.8 \mathrm{~Hz}), 2.13(3 \mathrm{H}, \mathrm{s}) .{ }^{13} \mathrm{C}-\mathrm{NMR}\left(\mathrm{CDCl}_{3}\right) \delta: 172.6,136.5$, 134.6, 133.7, 129.2, 129.0, 128.7, 128.6, 127.8, 126.5, 123.5, 77.0, 48.7, 20.5. MS (EI $\left.{ }^{+}\right) \mathrm{m} / z: 281\left(\mathrm{M}^{+}, 7\right), 91(100)$. HR-MS $m / z: 281.1417$ (Calcd for $\left.\mathrm{C}_{18} \mathrm{H}_{19} \mathrm{NO}_{2}: 281.1416\right)$.

$\mathrm{N}$-(4-Chlorocinnamyl)- $\mathrm{N}$-(benzyloxy)benzamide (11Ab) As a colorless oil: IR $\left(\mathrm{CHCl}_{3}\right) \mathrm{cm}^{-1}: 1637,1492,1450 .{ }^{1} \mathrm{H}-\mathrm{NMR}\left(\mathrm{CDCl}_{3}\right) \delta: 7.67(2 \mathrm{H}$, d, $J=7.0 \mathrm{~Hz}), 7.48(1 \mathrm{H}, \mathrm{t}, J=7.3 \mathrm{~Hz}), 7.44-7.20(9 \mathrm{H}, \mathrm{m}), 7.07(2 \mathrm{H}, \mathrm{d}$, $J=6.7 \mathrm{~Hz}), 6.54(1 \mathrm{H}, \mathrm{d}, J=15.9 \mathrm{~Hz}), 6.28(1 \mathrm{H}, \mathrm{dt}, J=15.9,6.1 \mathrm{~Hz}), 4.71$ $(2 \mathrm{H}, \mathrm{s}), 4.48(2 \mathrm{H}, \mathrm{d}, J=6.1 \mathrm{~Hz}) .{ }^{13} \mathrm{C}-\mathrm{NMR}\left(\mathrm{CDCl}_{3}\right) \delta: 170.2,134.9,134.3$, $134.2,133.5,132.6,130.6,129.5,128.8,128.7,128.5,128.3,128.1,127.7$, 124.1, 76.9, 50.3. MS $\left(\mathrm{EI}^{+}\right) \mathrm{m} / z: 377\left(\mathrm{M}^{+}, 0.5\right), 105$ (100). HR-MS $m / z$ : 377.1184 (Calcd for $\mathrm{C}_{23} \mathrm{H}_{20} \mathrm{ClNO}_{2}: 377.1188$ ).

$N$-(4-Fluorocinnamyl)- $N$-(benzyloxy)benzamide (11Ac) As a colorless oil: IR $\left(\mathrm{CHCl}_{3}\right) \mathrm{cm}^{-1}: 1636,1508,1450 .{ }^{1} \mathrm{H}-\mathrm{NMR}\left(\mathrm{CDCl}_{3}\right) \delta: 7.67(2 \mathrm{H}$, d, $J=7.0 \mathrm{~Hz}), 7.50-7.22(8 \mathrm{H}, \mathrm{m}), 7.11-6.97(4 \mathrm{H}, \mathrm{m}), 6.56(1 \mathrm{H}, \mathrm{d}, J=15.9$ $\mathrm{Hz}), 6.21(1 \mathrm{H}, \mathrm{dt}, J=15.9,6.1 \mathrm{~Hz}), 4.71(2 \mathrm{H}, \mathrm{s}), 4.47(2 \mathrm{H}, \mathrm{d}, J=6.1 \mathrm{~Hz})$. ${ }^{13} \mathrm{C}-\mathrm{NMR}\left(\mathrm{CDCl}_{3}\right) \delta: 170.3,162.5(\mathrm{~d}, J=247 \mathrm{~Hz}), 134.4,134.2,132.7$, $132.6,130.6,129.5,128.8,128.5,128.3,128.1$ (2C), 123.1, 115.5 (d, $J=22 \mathrm{~Hz}), 77.0,50.3$. MS $\left(\mathrm{EI}^{+}\right) \mathrm{m} / z: 361\left(\mathrm{M}^{+}, 0.5\right), 105(100)$. HR-MS $m / z$ : 361.1477 (Calcd for $\mathrm{C}_{23} \mathrm{H}_{20} \mathrm{FNO}_{2}: 361.1478$ ).

$N$-(Benzyloxy)- $N$-((E)-3-(naphthalen-1-yl)allyl)benzamide (11Ad) As a colorless oil: IR $\left(\mathrm{CHCl}_{3}\right) \mathrm{cm}^{-1}: 1637,1496,1450 .{ }^{1} \mathrm{H}-\mathrm{NMR}\left(\mathrm{CDCl}_{3}\right) \delta: 8.06$ $(1 \mathrm{H}, \mathrm{d}, J=8.9 \mathrm{~Hz}), 7.84(1 \mathrm{H}, \mathrm{d}, J=9.2 \mathrm{~Hz}), 7.78(1 \mathrm{H}, \mathrm{d}, J=7.9 \mathrm{~Hz}), 7.70$ $(2 \mathrm{H}, \mathrm{d}, J=7.0 \mathrm{~Hz}), 7.58(1 \mathrm{H}, \mathrm{d}, J=7.0 \mathrm{~Hz}), 7.53-7.32(7 \mathrm{H}, \mathrm{m}), 7.30-7.20$ $(3 \mathrm{H}, \mathrm{m}), 7.10(2 \mathrm{H}, \mathrm{d}, J=7.0 \mathrm{~Hz}), 6.34(1 \mathrm{H}, \mathrm{dt}, J=15.6,6.1 \mathrm{~Hz}), 4.76(2 \mathrm{H}, \mathrm{s})$, $4.61(2 \mathrm{H}, \mathrm{d}, J=6.1 \mathrm{~Hz}) .{ }^{13} \mathrm{C}-\mathrm{NMR}\left(\mathrm{CDCl}_{3}\right) \delta: 170.3,134.5,134.3,134.2$, 133.6, 131.3, 131.1, 130.6, 129.4, 128.8, 128.5 (2C), 128.3, 128.2, 128.1,

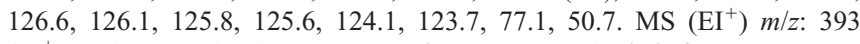
$\left(\mathrm{M}^{+}, 0.7\right), 105$ (100). HR-MS $\mathrm{m} / z$ : 393.1734 (Calcd for $\mathrm{C}_{27} \mathrm{H}_{23} \mathrm{NO}_{2}$ : 393.1729).
$(S)-N, N$-Dibenzyl-1-phenylprop-2-en-1-amine (18A) A colorless oil: IR $\left(\mathrm{CHCl}_{3}\right) \mathrm{cm}^{-1}: 1493,1451 .{ }^{1} \mathrm{H}-\mathrm{NMR}\left(\mathrm{CDCl}_{3}\right) \delta: 7.48(2 \mathrm{H}, \mathrm{d}, J=7.6 \mathrm{~Hz})$, $7.40(4 \mathrm{H}, \mathrm{d}, J=7.3 \mathrm{~Hz}), 7.35-7.15(9 \mathrm{H}, \mathrm{m}), 6.08$ (1H, ddd, $J=17.1,10.1$, $8.5 \mathrm{~Hz}), 5.44(1 \mathrm{H}, \mathrm{d}, J=10.1 \mathrm{~Hz}), 5.20(1 \mathrm{H}, \mathrm{d}, J=17.1 \mathrm{~Hz}), 4.27(1 \mathrm{H}, \mathrm{d}$, $J=8.5 \mathrm{~Hz}), 3.66(2 \mathrm{H}, \mathrm{d}, J=14.0 \mathrm{~Hz}), 3.53(2 \mathrm{H}, \mathrm{d}, J=14.0 \mathrm{~Hz}) .{ }^{13} \mathrm{C}-\mathrm{NMR}$ $\left(\mathrm{CDCl}_{3}\right) \delta: 141.5,140.1,135.4,128.7,128.3,128.2(2 \mathrm{C}), 127.0,126.8$, 119.4, 65.2, 53.6. MS (EI $\left.{ }^{+}\right) m / z: 313\left(\mathrm{M}^{+}, 22\right), 117$ (100). HR-MS $m / z$ : 313.1824 (Calcd for $\mathrm{C}_{23} \mathrm{H}_{23} \mathrm{~N}: 313.1830$ ). HPLC (Chiralcel OJ-H, hexane/2propanol $=95 / 5,0.5 \mathrm{ml} / \mathrm{min} 254 \mathrm{~nm}) t_{\mathrm{R}}(S)=10.8 \mathrm{~min}, t_{\mathrm{R}}(R)=14.0 \mathrm{~min}$. A sample of $95 \%$ ee $(S)$ by HPLC analysis gave $[\alpha]_{\mathrm{D}}^{31}-111.0(c=1.06$, $\mathrm{CHCl}_{3}$ ).

$(S)$ - $N$-Benzyl-1-phenylprop-2-en-1-amine $(\mathbf{1 8 B})^{42)}$ A colorless oil: IR $\left(\mathrm{CHCl}_{3}\right) \mathrm{cm}^{-1}: 3330,1493,1453 .{ }^{1} \mathrm{H}-\mathrm{NMR}\left(\mathrm{CDCl}_{3}\right) \delta: 7.38-7.22(10 \mathrm{H}$, m), $5.95(1 \mathrm{H}, \mathrm{ddd}, J=17.4,10.1,7.0 \mathrm{~Hz}), 5.22(1 \mathrm{H}, \mathrm{d}, J=17.4 \mathrm{~Hz}), 5.12$ $(1 \mathrm{H}, \mathrm{d}, J=10.1 \mathrm{~Hz}), 4.22(1 \mathrm{H}, \mathrm{d}, J=7.0 \mathrm{~Hz}), 3.74(1 \mathrm{H}, \mathrm{d}, J=13.1 \mathrm{~Hz}), 3.71$ $(1 \mathrm{H}, \mathrm{d}, J=13.1 \mathrm{~Hz}), 1.66(1 \mathrm{H}, \mathrm{brs}) .{ }^{13} \mathrm{C}-\mathrm{NMR}\left(\mathrm{CDCl}_{3}\right) \delta: 142.8,141.0$, $140.4,128.6,128.4,128.2,127.4,127.2,126.9,115.2,65.1,51.2$. MS $\left(\mathrm{FAB}^{+}\right) \mathrm{m} / z: 224\left(\mathrm{M}+\mathrm{H}^{+}, 81\right), 117$ (100). HR-MS $m / z$ : 224.1443 (Calcd for $\mathrm{C}_{16} \mathrm{H}_{18} \mathrm{~N}$ : 224.1439). HPLC (Chiralcel OD-H, hexane/2-propanol=99/1, $0.3 \mathrm{ml} / \mathrm{min} 254 \mathrm{~nm}) t_{\mathrm{R}}(S)=25.5 \mathrm{~min}, t_{\mathrm{R}}(R)=21.7 \mathrm{~min}$. A sample of $94 \%$ ee $(S)$ by HPLC analysis gave $[\alpha]_{\mathrm{D}}^{29}+5.0\left(c=1.0, \mathrm{CHCl}_{3}\right)$.

(S)- $N$-Benzyl- $N$-(benzyloxy)-1-phenylprop-2-en-1-amine (18C) A colorless oil: IR $\left(\mathrm{CHCl}_{3}\right) \mathrm{cm}^{-1}: 1494,1454 .{ }^{1} \mathrm{H}-\mathrm{NMR}\left(\mathrm{CDCl}_{3}\right) \delta: 7.48(2 \mathrm{H}, \mathrm{d}$, $J=7.3 \mathrm{~Hz}), 7.43-7.13(11 \mathrm{H}, \mathrm{m}), 6.79(2 \mathrm{H}, \mathrm{br} \mathrm{m}), 6.27(1 \mathrm{H}, \mathrm{m}), 5.26(1 \mathrm{H}, \mathrm{d}$, $J=17.4 \mathrm{~Hz}), 5.23(1 \mathrm{H}, \mathrm{d}, J=10.1 \mathrm{~Hz}), 4.29(1 \mathrm{H}, \mathrm{d}, J=8.5 \mathrm{~Hz}), 4.15(1 \mathrm{H}$, br m), $4.00(1 \mathrm{H}$, brd, $J=9.2 \mathrm{~Hz}), 3.89(1 \mathrm{H}, \quad$ brm $), 3.77(1 \mathrm{H}, \quad$ br d, $J=12.5 \mathrm{~Hz}) .{ }^{13} \mathrm{C}-\mathrm{NMR}\left(\mathrm{CDCl}_{3}\right) \delta: 141.4,138.3,138.0,136.7,130.1,129.2$, $128.5,128.4,128.1,127.8,127.4,127.2,117.6,76.8,75.5,60.8$. One carbon peak was missing due to overlapping. MS $\left(\mathrm{EI}^{+}\right) \mathrm{m} / \mathrm{z}: 329\left(\mathrm{M}^{+}, 3\right), 117(100)$. HR-MS $m / z$ : 329.1782 (Calcd for $\mathrm{C}_{23} \mathrm{H}_{23} \mathrm{NO}$ : 329.1780). HPLC (Chiralcel AD-H, hexane $/ 2$-propanol $=95 / 5,0.5 \mathrm{ml} / \mathrm{min}, 254 \mathrm{~nm}) t_{\mathrm{R}}(S)=7.1 \mathrm{~min}, t_{\mathrm{R}}$ $(R)=7.6 \mathrm{~min}$. A sample of $71 \%$ ee $(S)$ by HPLC analysis gave $[\alpha]_{\mathrm{D}}^{28}+15.2$ $\left(c=0.82, \mathrm{CHCl}_{3}\right)$.

(E)- $N$-Benzyl- $N$-(benzyloxy)-3-phenylprop-2-en-1-amine (19C) A colorless oil: IR $\left(\mathrm{CHCl}_{3}\right) \mathrm{cm}^{-1}: 1495,1452 .{ }^{1} \mathrm{H}-\mathrm{NMR}\left(\mathrm{CDCl}_{3}\right) \delta: 7.43-$ $7.08(15 \mathrm{H}, \mathrm{m}), 6.56(1 \mathrm{H}, \mathrm{d}, J=16.2 \mathrm{~Hz}), 6.36(1 \mathrm{H}, \mathrm{dt}, J=16.2,7.0 \mathrm{~Hz}), 4.44$ $(2 \mathrm{H}, \mathrm{br} \mathrm{s}), 3.89$ (2H. s), $3.56(2 \mathrm{H}, \mathrm{d}, J=7.0 \mathrm{~Hz}) .{ }^{13} \mathrm{C}-\mathrm{NMR}\left(\mathrm{CDCl}_{3}\right) \delta: 137.7$, 137.1 (2C), 133.6, 129.9, 129.1, 128.6, 128.2 (2C), 127.8, 127.5, 127.3, 126.4, 125.8, 76.0, 62.8, 60.9. MS (EI $\left.{ }^{+}\right) \mathrm{m} / z: 329\left(\mathrm{M}^{+}, 5\right), 117$ (100). HRMS $m / z: 329.1773$ (Calcd for $\mathrm{C}_{23} \mathrm{H}_{23} \mathrm{NO}: 329.1780$ ).

Enantioselective Allylic Substitution with $\boldsymbol{p}$-Anisidine A mixture of 20 ( $40.0 \mathrm{mg}, 0.325 \mathrm{mmol})$ and $\mathrm{CsOH} \cdot \mathrm{H}_{2} \mathrm{O}(54.5 \mathrm{mg}, 0.325 \mathrm{mmol})$ in $\mathrm{CH}_{2} \mathrm{Cl}_{2}$ $(1.0 \mathrm{ml})$ was stirred under argon atmosphere at $20^{\circ} \mathrm{C}$ for $10 \mathrm{~min}$. To the reaction mixture was added a solution of allylic phosphate 10a $(132 \mathrm{mg}$, $0.487 \mathrm{mmol})$, pybox $12(9.60 \mathrm{mg}, 0.0260 \mathrm{mmol})$ and $[\mathrm{IrCl}(\mathrm{cod})]_{2}(8.73 \mathrm{mg}$, $0.0130 \mathrm{mmol})$ in $\mathrm{CH}_{2} \mathrm{Cl}_{2}(1.0 \mathrm{ml})$ at $-20^{\circ} \mathrm{C}$. After the reaction was completed, the reaction mixture was diluted with water and then extracted with AcOEt. The organic phase was dried over $\mathrm{MgSO}_{4}$ and concentrated at reduced pressure. The ratio of products was determined by ${ }^{1} \mathrm{H}-\mathrm{NMR}$ analysis of crude products. Purification of the residue by preparative TLC (hexane: $\mathrm{AcOEt}=10: 1,2$-fold development) afforded the products 21 (64.8 mg, 84\%), 22 $\mathbf{2}^{89)}(7.3 \mathrm{mg}, 9 \%)$ and $\mathbf{2 3}^{89)}(8.1 \mathrm{mg}, 10 \%)$.

4-Methoxy- $N$ - $((S)-1 \text {-phenylallyl)benzenamine (21) })^{91)}$ A colorless oil: IR $\left(\mathrm{CHCl}_{3}\right) \mathrm{cm}^{-1}: 1512,1457 .{ }^{1} \mathrm{H}-\mathrm{NMR}\left(\mathrm{CDCl}_{3}\right) \delta: 7.42-7.21(5 \mathrm{H}, \mathrm{m})$, $6.72(2 \mathrm{H}, \mathrm{d}, J=8.9 \mathrm{~Hz}), 6.55(2 \mathrm{H}, \mathrm{d}, J=8.9 \mathrm{~Hz}), 6.02(1 \mathrm{H}, \mathrm{ddd}, J=17.1$, $10.1,5.8 \mathrm{~Hz}), 5.25(1 \mathrm{H}, \mathrm{d}, J=17.1 \mathrm{~Hz}), 5.19(1 \mathrm{H}, \mathrm{d}, J=10.1 \mathrm{~Hz}), 4.85(1 \mathrm{H}$, $\mathrm{d}, J=5.8 \mathrm{~Hz}), 3.70(3 \mathrm{H}, \mathrm{s}) .{ }^{13} \mathrm{C}-\mathrm{NMR}\left(\mathrm{CDCl}_{3}\right) \delta: 152.3,142.1,141.3,139.5$,

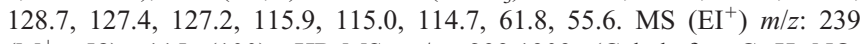
$\left(\mathrm{M}^{+}, 58\right), 115(100)$. HR-MS m/z: 239.1303 (Calcd for $\mathrm{C}_{16} \mathrm{H}_{17} \mathrm{NO}$ : 239.1310). HPLC (Chiralcel AD-H, hexane $/ 2$-propanol $=90 / 10,0.5 \mathrm{ml} / \mathrm{min}$ $254 \mathrm{~nm}) t_{\mathrm{R}}(S)=15.8 \mathrm{~min}, t_{\mathrm{R}}(R)=13.8 \mathrm{~min}$. A sample of $88 \%$ ee $(S)$ by HPLC analysis gave $[\alpha]_{\mathrm{D}}^{28}-12.1\left(c=0.95, \mathrm{CHCl}_{3}\right)$.

Enantioselective Allylic Substitution with 4-Methoxyphenol A mixture of $24(40.0 \mathrm{mg}, 0.322 \mathrm{mmol})$ and $\mathrm{K}_{2} \mathrm{CO}_{3}(44.5 \mathrm{mg}, 0.322 \mathrm{mmol})$ in $\mathrm{CH}_{2} \mathrm{Cl}_{2}(1.0 \mathrm{ml})$ was stirred under argon atmosphere at $20^{\circ} \mathrm{C}$ for $10 \mathrm{~min}$. To the reaction mixture was added a solution of allylic phosphate 10a $(131 \mathrm{mg}$, $0.483 \mathrm{mmol})$, pybox $12(9.52 \mathrm{mg}, 0.0258 \mathrm{mmol})$ and $[\mathrm{IrCl}(\mathrm{cod})]_{2}(8.66 \mathrm{mg}$, $0.0129 \mathrm{mmol})$ in $\mathrm{CH}_{2} \mathrm{Cl}_{2}(1.0 \mathrm{ml})$ at $-20^{\circ} \mathrm{C}$. After the reaction was completed, the reaction mixture was diluted with water and then extracted with AcOEt. The organic phase was dried over $\mathrm{MgSO}_{4}$ and concentrated at reduced pressure. The ratio of products was determined by ${ }^{1} \mathrm{H}-\mathrm{NMR}$ analysis of crude products. Purification of the residue by preparative TLC (hexane: $\mathrm{AcOEt}=15: 1,2$-fold development) afforded the products 25 (62.4 $\mathrm{mg}, 81 \%$ ) and $\mathbf{2 6}^{90)}(5.5 \mathrm{mg}, 7 \%)$. 


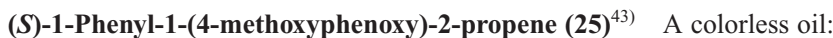
IR $\left(\mathrm{CHCl}_{3}\right) \mathrm{cm}^{-1}: 1505,1458 .{ }^{1} \mathrm{H}-\mathrm{NMR}\left(\mathrm{CDCl}_{3}\right) \delta: 7.42-7.25(5 \mathrm{H}, \mathrm{m})$, $6.86(2 \mathrm{H}, \mathrm{d}, J=9.2 \mathrm{~Hz}), 6.76(2 \mathrm{H}, \mathrm{d}, J=9.2 \mathrm{~Hz}), 6.09(1 \mathrm{H}, \mathrm{ddd}, J=17.1$, $10.4,5.8 \mathrm{~Hz}), 5.52(1 \mathrm{H}, \mathrm{d}, J=5.8 \mathrm{~Hz}), 5.32(1 \mathrm{H}, \mathrm{d}, J=17.1 \mathrm{~Hz}), 5.24(1 \mathrm{H}, \mathrm{d}$, $J=10.4 \mathrm{~Hz}), 3.73(3 \mathrm{H}, \mathrm{s}) .{ }^{13} \mathrm{C}-\mathrm{NMR}\left(\mathrm{CDCl}_{3}\right) \delta: 154.1,152.1,140.3,138.2$, $128.6,127.8,126.7,117.5,116.5,114.5,81.9$, 55.6. $\mathrm{MS}\left(\mathrm{EI}^{+}\right) \mathrm{m} / z: 240$ $\left(\mathrm{M}^{+}, 87\right), 136$ (100). HR-MS $m / z: 240.1154$ (Calcd for $\mathrm{C}_{16} \mathrm{H}_{16} \mathrm{O}_{2}$ : 240.1150). HPLC (Chiralcel OD-H, hexane/2-propanol=95/5, $0.5 \mathrm{ml} / \mathrm{min}$ $254 \mathrm{~nm}) t_{\mathrm{R}}(S)=21.6 \mathrm{~min}, t_{\mathrm{R}}(R)=19.2 \mathrm{~min}$. A sample of $72 \%$ ee $(S)$ by HPLC analysis gave $[\alpha]_{\mathrm{D}}^{31}-5.8\left(c=1.4, \mathrm{CHCl}_{3}\right)$.

Reduction of 9Aa into 13 To a solution of 9Aa $(40.0 \mathrm{mg}, 0.116 \mathrm{mmol})$ in $\mathrm{AcOH}-\mathrm{H}_{2} \mathrm{O}(1.0 \mathrm{ml}, 1: 1, \mathrm{v} / \mathrm{v})$ was added $\mathrm{Zn}$ powder $(305 \mathrm{mg}, 4.66$ $\mathrm{mmol}$ ) at $20^{\circ} \mathrm{C}$. After being stirred at $60^{\circ} \mathrm{C}$ for $20 \mathrm{~h}$, the reaction mixture was diluted with saturated $\mathrm{NaHCO}_{3}$ and then extracted with $\mathrm{CHCl}_{3}$. The organic phase was dried over $\mathrm{MgSO}_{4}$ and concentrated at reduced pressure. Purification of the residue by preparative TLC (hexane: $\mathrm{AcOEt}=8: 1$ ) afforded the product $13^{85)}(21.6 \mathrm{mg}, 78 \%)$ as a white solid. IR $\left(\mathrm{CHCl}_{3}\right) \mathrm{cm}^{-1}$ : 3445, 1662, 1510, 1482. ${ }^{1} \mathrm{H}-\mathrm{NMR}\left(\mathrm{CDCl}_{3}\right) \delta: 7.79(2 \mathrm{H}, \mathrm{d}, J=7.0 \mathrm{~Hz})$, $7.51-7.27(8 \mathrm{H}, \mathrm{m}), 6.44(1 \mathrm{H}, \mathrm{br} \mathrm{d}, J=6.7 \mathrm{~Hz}), 6.11(1 \mathrm{H}, \mathrm{ddd}, J=17.4,10.2$ $5.5 \mathrm{~Hz}), 5.85(1 \mathrm{H}$, br m), $5.32(1 \mathrm{H}, \mathrm{d}, J=10.2 \mathrm{~Hz}), 5.29(1 \mathrm{H}, \mathrm{d}, J=17.4 \mathrm{~Hz})$ ${ }^{13} \mathrm{C}-\mathrm{NMR}\left(\mathrm{CDCl}_{3}\right) \delta: 166.6,140.6,137.2,134.4,131.6,128.9,128.6,127.8$, 127.3, 127.0, 116.2, 55.5. MS (EI $\left.{ }^{+}\right) \mathrm{m} / z: 237\left(\mathrm{M}^{+}, 35\right), 105$ (100). HR-MS $m / z: 237.1160$ (Calcd for $\left.\mathrm{C}_{16} \mathrm{H}_{15} \mathrm{NO}: 237.1154\right) .[\alpha]_{\mathrm{D}}^{29}-70.6(c=1.0$, $\mathrm{CHCl}_{3}$ ).

Conversion of 9Ca into 14 A mixture of 9 Ca $(40.0 \mathrm{mg}, 0.136 \mathrm{mmol})$ and 2nd Grubbs' Ru-catalyst 15 (11.6 mg, $0.0136 \mathrm{mmol})$ in $\mathrm{CH}_{2} \mathrm{Cl}_{2}(8 \mathrm{ml})$ was stirred under argon atmosphere at reflux for $12 \mathrm{~h}$. After the reaction was completed, the reaction mixture was diluted with water and then extracted with $\mathrm{CH}_{2} \mathrm{Cl}_{2}$. The organic phase was dried over $\mathrm{MgSO}_{4}$ and concentrated at reduced pressure. Purification of the residue by preparative TLC (hexane : AcOEt =7:1) afforded the product $14(31.2 \mathrm{mg}, 87 \%)$ as colorless oil. IR $\left(\mathrm{CHCl}_{3}\right) \mathrm{cm}^{-1}: 1634,1494,1449,1410 .{ }^{1} \mathrm{H}-\mathrm{NMR}\left(\mathrm{CDCl}_{3}\right) \delta: 7.67$ $(2 \mathrm{H}, \mathrm{d}, J=7.3 \mathrm{~Hz}), 7.51(2 \mathrm{H}, \mathrm{d}, J=7.3 \mathrm{~Hz}), 7.45-7.30(6 \mathrm{H}, \mathrm{m}), 6.07-6.01$ $(3 \mathrm{H}, \mathrm{m}), 4.52(1 \mathrm{H}$, brd, $J=15.6 \mathrm{~Hz}), 4.29(1 \mathrm{H}, \mathrm{dd}, J=15.6,3.0 \mathrm{~Hz}) .{ }^{13} \mathrm{C}-$ NMR $\left(\mathrm{CDCl}_{3}\right) \delta: 168.8,138.5,133.8,130.8,128.6,128.5,128.0,127.9$ $125.7,123.6,69.6,55.1$. One carbon peak was missing due to overlapping. MS (EI $\left.{ }^{+}\right) m / z: 265\left(\mathrm{M}^{+}, 2\right), 105$ (100). HR-MS m/z: 265.1095 (Calcd for $\mathrm{C}_{17} \mathrm{H}_{15} \mathrm{NO}_{2}$ : 265.1103). $[\alpha]_{\mathrm{D}}^{30}+405\left(c=1.08, \mathrm{CHCl}_{3}\right)$.

Reduction of 14 into 16 To a solution of $14(47.0 \mathrm{mg}, 0.177 \mathrm{mmol})$ in $\mathrm{AcOH}-\mathrm{H}_{2} \mathrm{O}(1.6 \mathrm{ml}, 1: 1, \mathrm{v} / \mathrm{v})$ was added $\mathrm{Zn}$ powder $(463 \mathrm{mg}, 7.09 \mathrm{mmol})$ at $20^{\circ} \mathrm{C}$. After being stirred at $60^{\circ} \mathrm{C}$ for $20 \mathrm{~h}$, the reaction mixture was diluted with saturated $\mathrm{NaHCO}_{3}$ and then extracted with $\mathrm{CHCl}_{3}$. The organic phase was dried over $\mathrm{MgSO}_{4}$ and concentrated at reduced pressure. Purification of the residue by preparative TLC (hexane : $\mathrm{AcOEt}=2: 1$ ) afforded the product $16(37.7 \mathrm{mg}, 80 \%)$ as colorless crystal. $\mathrm{mp} 128-130^{\circ} \mathrm{C}$ (AcOEt/hexane). IR $\left(\mathrm{CHCl}_{3}\right) \mathrm{cm}^{-1}: 3438,1652,1512,1481 .{ }^{1} \mathrm{H}-\mathrm{NMR}\left(\mathrm{CDCl}_{3}\right) \delta: 7.77(2 \mathrm{H}$, d, $J=7.3 \mathrm{~Hz}), 7.50(1 \mathrm{H}, \mathrm{t}, J=7.3 \mathrm{~Hz}), 7.45-7.31(7 \mathrm{H}, \mathrm{m}), 6.73(1 \mathrm{H}, \mathrm{brs})$, $6.15(1 \mathrm{H}$, brt,$J=8.6 \mathrm{~Hz}), 5.99(1 \mathrm{H}$, br m), $5.74(1 \mathrm{H}, \mathrm{t}, J=10.4 \mathrm{~Hz}), 4.55$ $(1 \mathrm{H}, \mathrm{dd}, J=13.0,8.1 \mathrm{~Hz}), 4.05(1 \mathrm{H}, \mathrm{dd}, J=13.0,6.0 \mathrm{~Hz}), 3.82(1 \mathrm{H}, \mathrm{br})$. ${ }^{13} \mathrm{C}-\mathrm{NMR}\left(\mathrm{CDCl}_{3}\right) \delta: 167.2,140.1,134.0,131.9,131.6,130.6,129.0,128.6$, 128.0, 127.0, 126.7, 57.9, 50.5. MS $\left(\mathrm{FAB}^{+}\right) 268\left(\mathrm{M}+\mathrm{H}^{+}, 94\right), 105(100)$ HR-MS $m / z$ : 268.1332 (Calcd for $\mathrm{C}_{17} \mathrm{H}_{18} \mathrm{NO}_{2}:$ 268.1337). $[\alpha]_{\mathrm{D}}^{23}+3.7$ $\left(c=0.7, \mathrm{CHCl}_{3}\right)$. Anal. Calcd for $\mathrm{C}_{17} \mathrm{H}_{18} \mathrm{NO}_{2}$ : C, 76.38; H, 6.41; N, 5.24. Found: C, 76.30; H, 6.60; N, 5.14.

Acknowledgements This work was supported in part by a Grant-in-Aid for Scientific Research (C) (H.M.) from the Ministry of Education, Culture, Sports, Science and Technology of Japan.

\section{References}

1) Johannsen M., Jorgensen K. A., Chem. Rev., 98, 1689-1708 (1998).

2) Helmchen G., J. Organomet. Chem., 576, 203-214 (1999).

3) Trost B. M., Crawley M. L., Chem. Rev., 103, 2921-2944 (2003).

4) Graening T., Schmalz H.-G., Angew. Chem. Int. Ed., 42, 2580-2584 (2003).

5) Trost B. M., J. Org. Chem., 69, 5813-5837 (2004)

6) Takemoto Y., Miyabe H., "Comprehensive Organometallic Chemistry," 3rd ed., Vol. 10.15, ed. by Crabtree R. H., Mingos D. M. P., Elsevier, Oxford, 2006, pp. 695-724.

7) Lu Z., Ma S., Angew. Chem. Int. Ed., 47, 258-297 (2008)

8) Takemoto Y., Miyabe H., "Catalytic Asymmetric Synthesis," 3rd ed., ed. by Ojima I., John Wiley \& Sons, U.K., 2010, pp. 227-267.

9) Miyabe H., Takemoto Y., Synlett, 2005, 1641-1655 (2005).

10) Miyabe H., Matsumura A., Yoshida K., Yamauchi M., Takemoto Y.,
Synlett, 2004, 2123-2126 (2004).

11) Miyabe H., Yoshida K., Reddy V. K., Matsumura A., Takemoto Y., J. Org. Chem., 70, 5630-5635 (2005).

12) Reddy V. K., Miyabe H., Yamauchi M., Takemoto Y., Tetrahedron, 64, 1040 - 1048 (2008)

13) Miyabe H., Yoshida K., Reddy V. K., Takemoto Y., J. Org. Chem., 74, 305-311 (2009).

14) Miyabe H., Matsumura A., Yoshida K., Takemoto Y., Tetrahedron, 65 , 4464-4470 (2009).

15) Murahashi S., Imada Y., Taniguchi Y., Kodera Y., Tetrahedron Lett., 29, 2973-2976 (1988)

16) Genet J.-P., Thorimbert S., Touzin A.-M., Tetrahedron Lett., 34, 1159-1162 (1993)

17) Miyabe H., Yoshida K., Yamauchi M., Takemoto Y., J. Org. Chem., 70, $2148-2153$ (2005)

18) Miyabe H., Yoshida K., Matsumura A., Yamauchi M., Takemoto Y., Synlett, 2003, 567-569 (2003).

19) Preliminary communication, see: Miyabe H., Matsumura A., Moriyama K., Takemoto Y., Org. Lett., 6, 4631-4634 (2004).

20) Hayashi T., Kishi K., Yamamoto A., Ito Y., Tetrahedron Lett., 31, $1743-1746$ (1990)

21) Trost B. M., Krische M. J., Radinov R., Zanoni G., J. Am. Chem. Soc., 118, 6297-6298 (1996).

22) Trost B. M., Toste F. D., J. Am. Chem. Soc., 121, 4545-4554 (1999).

23) You S.-L., Zhu X.-Z., Luo Y.-M., Hou X.-L., Dai L.-X., J. Am. Chem. Soc., 123, 7471-7472 (2001).

24) Lüssem, B. J., Gais, H.-J., J. Am. Chem. Soc., 125, 6066-6067 (2003).

25) Leahy D. K., Evans P. A., "Modern Rhodium-Catalyzed Organic Reactions," Chap. 10, ed. by Evans P. A., Wiley-VCH, Weinheim, 2005, pp. $191-214$

26) Evans P. A., Robinson J. E., Nelson J. P., J. Am. Chem. Soc., 121, 6761-6762 (1999)

27) Evans P. A., Leahy D. K., J. Am. Chem. Soc., 122, 5012-5013 (2000).

28) Evans P. A., Robinson J. E., Moffett K. K., Org. Lett., 3, 3269-3271 (2001).

29) Evans P. A., Leahy D. K., J. Am. Chem. Soc., 124, 7882-7883 (2002)

30) Evans P. A., Leahy D. K., Andrews W. J., Uraguchi D., Angew. Chem Int. Ed., 43, 4788-4791 (2004).

31) Evans P. A., Lai K. W., Zhang H.-R., Huffman J. C., Chem. Commun., 2006, 844-846 (2006).

32) Evans P. A., Clizbe E. A., J. Am. Chem. Soc., 131, 8722-8723 (2009).

33) Vrieze D. C., Hoge G. S., Hoerter P. Z., Haitsma J. T. V., Samas B. M., Org. Lett., 11, 3140-3142 (2009).

34) Takeuchi R., Synlett, 2002, 1954-1965 (2002).

35) Takeuchi R., Ue N., Tanabe K., Yamashita K., Shiga N., J. Am. Chem Soc., 123, 9525-9534 (2001).

36) Takeuchi R., Shiga N., Org. Lett., 1, 265-268 (1999).

37) Takeuchi R., Kezuka S., Synthesis, 2006, 3349-3366 (2006).

38) Helmchen G., Dahnz A., Dübon P., Schelwies M., Weihofen R., Chem. Commun., 2007, 675-691 (2007).

39) Mbaye M. D., Renaud J.-L., Demerseman B., Bruneau C., Chem. Commun., 2004, 1870-1871 (2004).

40) Onitsuka K., Okuda H., Sasai H., Angew. Chem. Int. Ed., 47, 1454 1457 (2008).

41) Austeri M., Linder D., Lacour J., Chem. Eur. J., 14, 5737-5741 (2008).

42) Ohmura T., Hartwig J. F., J. Am. Chem. Soc., 124, 15164-15165 (2002).

43) López F., Ohmura T., Hartwig J. F., J. Am. Chem. Soc., 125, 3426 3427 (2003).

44) Kiener C. A., Shu C., Incarvito C., Hartwig J. F., J. Am. Chem. Soc., 125, 14272-14273 (2003).

45) Shu C., Hartwig J. F., Angew. Chem. Int. Ed., 43, $4794-4797$ (2004).

46) Shu C., Leitner A., Hartwig J. F., Angew. Chem. Int. Ed., 43, 47974800 (2004).

47) Leitner A., Shekhar S., Pouy M. J., Hartwig J. F., J. Am. Chem. Soc., 127, 15506-15514 (2005).

48) Leitner A., Shu C., Hartwig J. F., Org. Lett., 7, 1093-1096 (2005).

49) Shekhar S., Trantow B., Leitner A., Hartwig J. F., J. Am. Chem. Soc., 128, 11770-11771 (2006).

50) Pouy M. J., Leitner A., Weix D. J., Ueno S., Hartwig J. F., Org. Lett., 9, 3949-3952 (2007).

51) Yamashita Y., Gopalarathnam A., Hartwig J. F., J. Am. Chem. Soc., 
129, 7508-7509 (2007)

52) Marković D., Hartwig J. F., J. Am. Chem. Soc., 129, 11680-11681 (2007).

53) Ueno S., Hartwig J. F., Angew. Chem. Int. Ed., 47, 1928-1931 (2008).

54) Stanley L. M., Hartwig J. F., J. Am. Chem. Soc., 131, 8971-8983 (2009).

55) Pouy M. J., Stanley L. M., Hartwig J. F., J. Am. Chem. Soc., 131, 11312-11313 (2009).

56) Ueda M., Hartwig J. F., Org. Lett., 12, $92-94$ (2010).

57) Stanley L. M., Bai C., Ueda M., Hartwig J. F., J. Am. Chem. Soc., 132, 8918 -8920 (2010).

58) Lipowsky G., Helmchen G., Chem. Commun., 2004, 116-117 (2004).

59) Welter C., Koch O., Lipowsky G., Helmchen G., Chem. Commun., 2004, 896-897 (2004)

60) Welter C., Dahnz A., Brunner B., Streiff S., Duebon P., Helmchen G., Org. Lett., 7, 1239-1242 (2005).

61) Weihofen R., Dahnz A., Tverskoy O., Helmchen G., Chem. Commun., 2005, 3541-3543 (2005).

62) Welter C., Moreno R. M., Streiff S., Helmchen G., Org. Biomol. Chem., 3, 3266-3268 (2005).

63) Weihofen R., Tverskoy O., Helmchen G., Angew. Chem. Int. Ed., 45, 5546 - 5549 (2006).

64) Spiess S., Berthold C., Weihofen R., Helmchen G., Org. Biomol. Chem., 5, 2357-2360 (2007).

65) Gnamm C., Franck G., Miller N., Stork T., Brödner K., Helmchen G., Synthesis, 2008, 3331-3350 (2008).

66) Gnamm C., Krauter C. M., Brödner K., Helmchen G., Chem. Eur. J., 15, 2050-2054 (2009)

67) Gnamm C., Brödner K., Krauter C. M., Helmchen G., Chem. Eur. J., 15, 10514-10532 (2009).

68) Farwick A., Helmchen G., Org. Lett., 12, 1108-1111 (2010).

69) Raskatov J. A., Spiess S., Gnamm C., Brödner K., Rominger F., Helmchen G., Chem. Eur. J., 16, 6601-6615 (2010).

70) Tissot-Croset K., Polet D., Alexakis A., Angew. Chem. Int. Ed., 43, $2426-2428$ (2004).
71) Polet D., Alexakis A., Org. Lett., 7, 1621-1624 (2005).

72) Polet D., Alexakis A., Tissot-Croset K., Corminboeuf C., Ditrich K., Chem. Eur. J., 12, 3596-3609 (2006).

73) Fischer C., Defieber C., Suzuki T., Carreira E. M., J. Am. Chem. Soc., 126, 1628-1629 (2004).

74) Lyothier I., Defieber C., Carreira E. M., Angew. Chem. Int. Ed., 45, 6204-6207 (2006)

75) Defieber C., Ariger M. A., Moriel P., Carreira E. M., Angew. Chem. Int. Ed., 46, 3139-3143 (2007).

76) Singh O. V., Han H., J. Am. Chem. Soc., 129, 774-775 (2007).

77) Singh O. V., Han H., Org. Lett., 9, 4801-4804 (2007).

78) Singh O. V., Han H., Tetrahedron Lett., 48, 7094-7098 (2007).

79) Nemoto T., Sakamoto T., Matsumoto T., Hamada Y., Tetrahedron Lett., 47, 8737-8740 (2006).

80) Kimura M., Uozumi Y., J. Org. Chem., 72, 707-714 (2007).

81) Bondzic B. P., Farwick A., Liebich J., Eilbracht P., Org. Biomol. Chem., 6, 3723-3731 (2008).

82) Xu Q.-L., Dai L.-X., You S.-L., Org. Lett., 12, 800-803 (2010).

83) He H., Liu W.-B., Dai L.-X., You S.-L., Angew. Chem. Int. Ed., 49, 1496-1499 (2010).

84) Xia J.-B., Liu W.-B., Wang T.-M., You S.-L., Chem. Eur. J., 16, 64426446 (2010).

85) Castagnolo D., Armaroli S., Corelli F., Botta M., Tetrahedron: Asymmetry, 15, 941-949 (2004).

86) Yoshioka E., Kohtani S., Miyabe H., Trend. Heterocyclic Chem., 14, $1-16$ (2009).

87) Kadota I., Shibuya A., Lutete L. M., Yamamoto Y., J. Org. Chem., 64, 4570- 4571 (1999)

88) Rehn S., Ofial A. R., Mayr H., Synthesis, 12, 1790-1796 (2003).

89) Yang S.-C., Hsu Y.-C., Gan K.-H., Tetrahedron, 62, 3949-3958 (2006).

90) Yatsumonji Y., Ishida Y., Tsubouchi A., Takeda T., Org. Lett., 9, 4603-4606 (2007).

91) Weiss M. E., Fischer D. F., Xin Z., Jautze S., Schweizer W. B., Peters R., Angew. Chem. Int. Ed., 45, 5694-5698 (2006). 